\title{
Nanomedicine applied to translational oncology: a future perspective on cancer treatment
}

\section{Short title: Nanomedicine applied to translational oncology}

Dr. Lisa Bregoli, PhD, Department of Clinical Medicine, Institute of Molecular Medicine (IMM), School of Medicine, Trinity College Dublin, Dublin 8, Ireland

Dr. Dania Movia, PhD, AMBER Centre and CRANN Institute, Trinity College Dublin, Dublin 2, Ireland

Mr. James D. Gavigan-Imedio, MSc, Institute of Protein Biochemistry, Consiglio Nazionale delle Ricerche (CNR), Napoli, Italy

Dr. Joanne Lysaght, PhD, Department of Surgery, Trinity Centre for Health Sciences, St. James's Hospital, Dublin 8.

Prof. John Reynolds, MD PhD, Department of Surgery, Trinity Centre for Health Sciences, St. James's Hospital, Dublin 8.

Dr. Adriele Prina-Mello, MSc PhD, Nanomedicine and Molecular Imaging Group, Department of Clinical Medicine, Institute of Molecular Medicine (IMM), School of Medicine, Trinity College Dublin, Dublin 8, Ireland; and AMBER Centre and CRANN Institute, Trinity College Dublin, Dublin 2, Ireland

\section{Corresponding author:}

Dr Adriele Prina-Mello, PhD, MSc, Room 0.75, Nanomedicine and Molecular Imaging Group, Department of Clinical Medicine, Institute of Molecular Medicine (IMM), School of Medicine, Trinity College Dublin, Dublin 8, St. James's Street, Dublin 8, Ireland.

T: +353-1-8963087, F: +353-1-8963037; E: prinamea@tcd.ie

Abstract word count: 141

Complete manuscript: 12,745

Number of figures: 5

Number of tables: 3

Number of references: 173

Funded: This work was partially supported by the EU FP7 NAMDIATREAM project (contract \# 246479), NANoREG project (contract \# 310584), MULTIFUN project (contract \# 262943) and Science Foundation Ireland (grant \#SFI/12/RC/2278).

Conflict of interest: The authors declare no competing financial interests. 


\section{ABSTRACT}

The high global incidence of cancer is associated with high rates of mortality and morbidity worldwide. By taking advantage of the properties of matter at the nanoscale, nanomedicine promises to develop innovative drugs with greater efficacy and less side effects than standard therapies.

Here, we discuss both clinically available anti-cancer nanomedicines and those en route to future clinical application. The properties, therapeutic value, advantages and limitations of these nanomedicine products are highlighted, with a focus on their increased performance versus conventional molecular anticancer therapies. The main regulatory challenges towards the translation of innovative, clinically effective nanotherapeutics are discussed, with a view to improving current approaches to the clinical management of cancer.

Ultimately, it becomes clear that the critical steps for clinical translation of nanotherapeutics require further interdisciplinary and international effort, where the whole stakeholder community is involved from bench to bedside.

\section{Keywords:}

Cancer therapeutics, nanomedicine, translational oncology, nanodrug design, nanomedicine regulation

\section{Abbreviations:}

EPR: enhanced permeability and retention

MPS: multinucleate phagocytic system

PEG: Polyethylene glycol

EMA: European Medicines Association

FDA: Food and Drug Administration

R\&D: Research and Development 


\section{Background}

The growing interest in the medical application of nanotechnology by academic and industrial researchers worldwide has led to the development of novel nanomedical platforms and nanodrugs, along with a substantial increase in government funding and venture-capital investment $(1,2)$. The latest are driven societally by the promise of significant improvements in the sensitivity, efficacy and safety of existing diagnostic and treatment strategies, as well as economically, by the attendant business opportunities that might arise from this emerging market.

Cancer nanomedicine, one of the most prominent areas in this field $(1,3)$, lies at the interface of different scientific disciplines including physics, chemistry, cancer research and clinical practice (Figure 1). Cancer nanomedicines are generally intended as miniaturized delivery systems, also termed nanocarriers, nanotherapeutics or nanodrugs, which aim at improving the therapeutic efficacy of currently available chemotherapeutic agents by combining them with a nanoscale delivery component. However, the overall picture of the potential use of nanomaterials in oncology also includes diagnostics, theranostics, medical devices and, more recently, therapeutics for personalized medicine (4-8). For instance, the use of nanomaterials can improve the target-specificity and/or tissue penetration of a diagnostic probe, thus allowing earlier detection of malignancy. Similarly, theranostics benefit from nanotechnology in the development of multifunctional clinical products that allow real-time tracking of the targeted delivery of therapeutics to the patient $(9,10)$.

Notably, the most advanced applications of nanomedicine currently available on the market are commercialized under the diagnostics umbrella. This is partly linked to the current lack of a specific regulatory framework for nano-therapeutics, a deficiency that leads to the first major regulatory dilemma posed by nanomedical products, classification. In fact, the development of nanomedicine has brought to multimodal hybrid structures that can act both as therapeutic and imaging agents and are in many cases 
developed as combination products. This combination of therapeutic and diagnostic properties falls into a grey area of regulatory classification, leading to the fact that European regulation for medical devices may be more applicable to "next-generation nanomedicines", than pharmaceutical regulation. The lack of clear and effective definitions outlining the field results in the fact that, in many cases, the nanotherapeutic approach falls under numerous different regulatory categories (3). Regulatory uncertainty is, in turn, also linked to scientific knowledge gaps about safety and long-term effects of nanomaterials, leading to inadequate standards and protocols for production scale-up and Inadequate standards and protocols for safe clinical use (11), thus creating barriers to public acceptance, investor interest and commercial approval (12). Hence, the nanomedicine market is currently paused at a critical stage where clear regulatory guidance is needed to provide clarity and legal certainty to industrial/academic R\&D, as well as healthcare stakeholders and society.

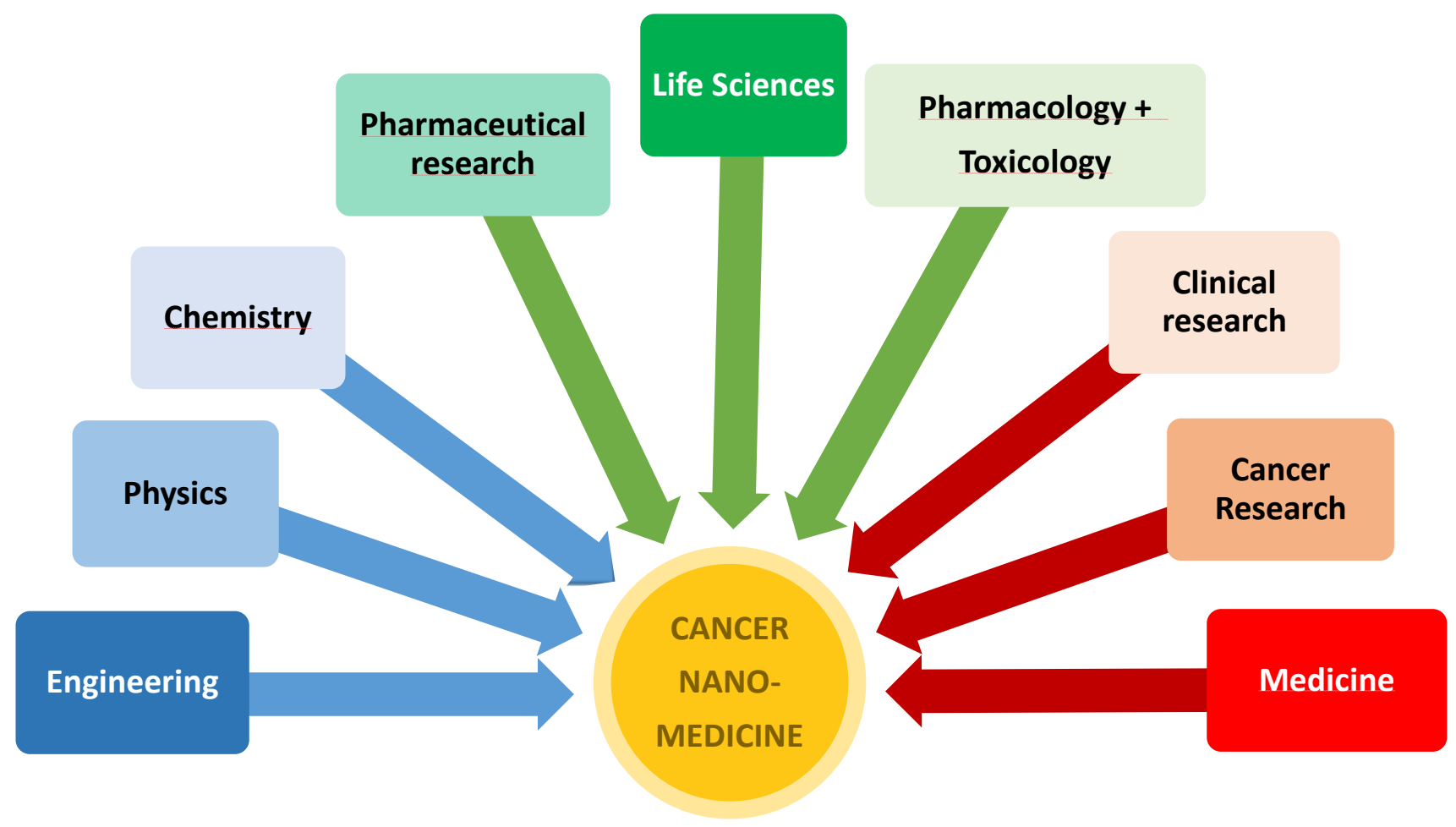


Figure 1. Multiple disciplines converge in cancer nanomedicine. As shown, the field requires integration of knowledge from many scientific disciplines.

Relevant challenges to nanomedicine translation also lie in the real difficulty of reaching an advanced interdisciplinary forum for discussion, where not only experts from several field from academia, but also clinicians and professionals from the pharmaceutical/healthcare industries and regulatory bodies can discuss ideas, issues and unmet needs(13). The complex challenges facing all stakeholders (Figure 2) will be described in this review with a particular emphasis on scientific and regulatory issues.

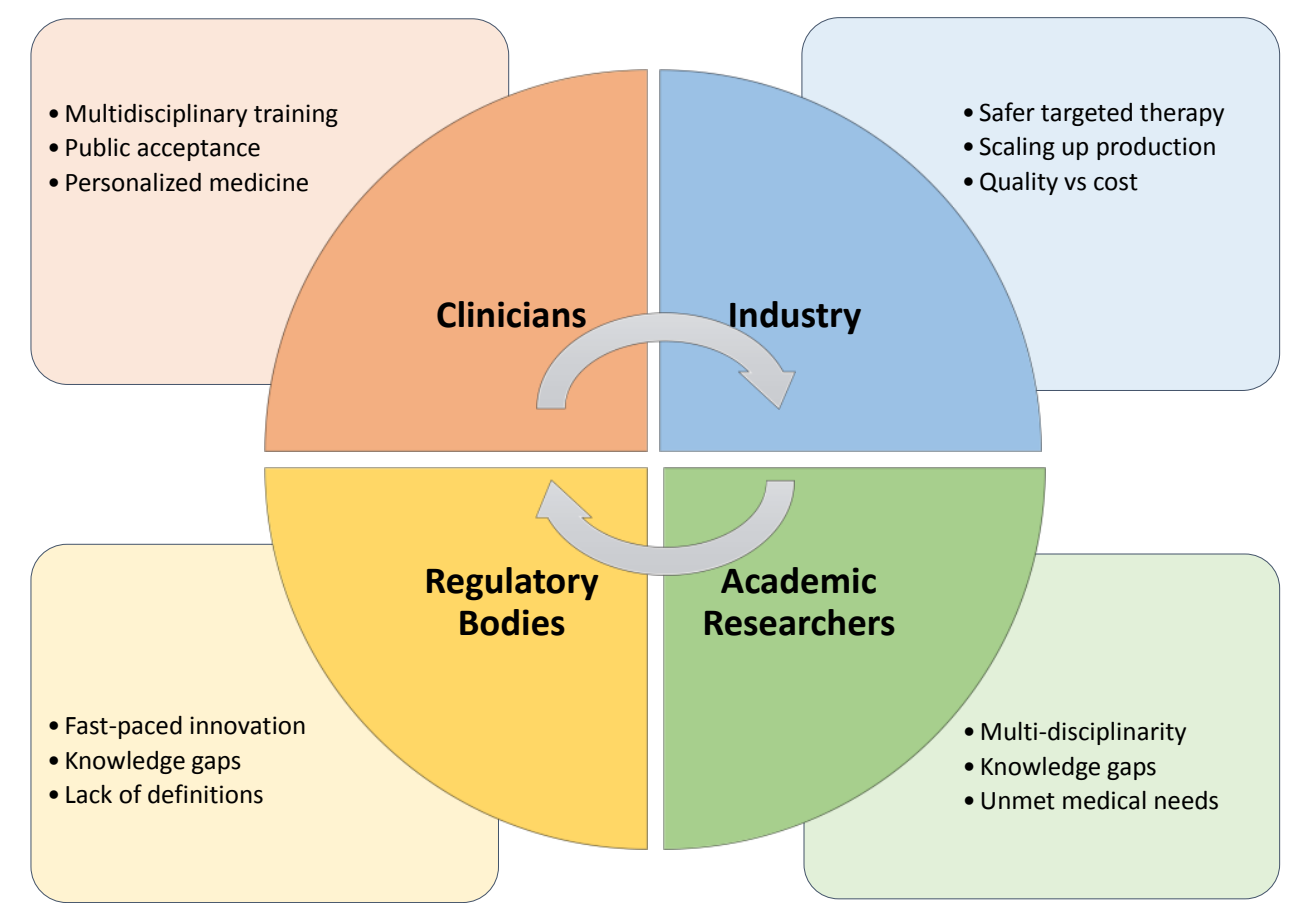

Figure 2. Stakeholders and Challenges. The main nanomedicine stakeholders (i.e., clinicians, patients, industry, regulatory bodies, and academic researchers) and the main challenges faced in the translation of nanomedicines. 
Keeping the breadth of cancer treatment application in mind, this review focuses on nanomedicines that are aimed at increasing the efficacy of currently available cancer therapies. The regulatory challenges of commercialization will also be discussed in light of the need for effective translation of "next-generation nanotherapeutics" from proven preclinical tools to clinically effective, marketable products.

\section{Design of nanomaterials for cancer therapeutic applications}

The medical use of manufactured nanomaterials in oncology is gaining ground rapidly, with various nanobased products already on the market and many progressing through different stages of the drug discovery pipeline. Nanomaterials designed for cancer therapy can be as diverse as micelles, liposomes, dendrimers, inorganic nanoparticles, carbon nanoparticles and nanotubes, nanodiamonds, nanoemulsions, viral nanocarriers, polymeric or peptide nanoparticles, and solid lipid nanoparticles; they can self-assemble or be directly synthesized into multicomponent and multifunctional systems $(7,14-23)$. Nanodrugs can be used as stand-alone cancer therapies, or they can be used as adjuvants or as part of a combinatorial therapy.

One of the most frequent applications of nanomaterials in oncology is to improve the performance of anti-cancer drugs in terms of bioavailability, safety and specificity, by taking advantage of the properties of nanoscale particulate. Chemotherapeutic drugs can be loaded onto nanomaterials via physical entrapment, adsorption through non-covalent interactions, or by covalent bonding to the nanomaterial surface via degradable or non-degradable bonds. The tunable size, shape, and surface characteristics of nanomaterials enable the production of nanomedicines with high stability, solubility, and compatibility in biological fluids, and the incorporation of both hydrophilic and hydrophobic substances. Accordingly, nanocarriers can improve the solubility of commercially available chemotherapeutic agents, thus 
extending their circulation half-life. The results are dramatic improvements in clinical infusion schedules and the patient's overall experience of the treatment.

Arguably, the most striking advantage of oncological nanomedicines is their capacity to enhance the efficacy of existing clinically approved anticancer drugs by increasing the efficiency and/or specificity of delivery to their sites of action. This is a direct consequence of the nanomaterials' size and surface properties, which enhance tissue penetration of its cargo, i.e. the drug. It is also linked to the fact that it is relatively easy to functionalize the surface of nanomaterials with biological targeting moieties, thus allowing specific delivery of chemotherapeutics to the malignant tissue, and thereby reducing toxicity in healthy tissue. Use of such nanocarriers has indeed been found to produce dramatic improvements in the therapeutic index of associated nano-drugs; mainly by increasing doses at the disease site, while reducing side effects $(5,24,25)$.

Another common but quite different use of nanomaterials for cancer treatment is that of inorganic particles for photothermal therapy (26). In this case, the nanoparticles are not used as drug-delivery systems; instead, they act directly as therapeutic agents by producing thermal damage in tumor tissue upon application of an external electromagnetic field. This approach avoids side effects arising from metabolic degradation of therapeutic compounds in the liver.

\section{Targeting mechanisms}

The uptake and accumulation of nanodrugs in cancerous tissue can occur through two mechanisms, usually referred to as "passive targeting" and "active targeting". Passive accumulation at the tumor site is thought to take place via a process known as Enhanced Permeability and Retention (EPR) effect. EPR is due to the increased vessel leakiness and impaired lymphatic function usually found in tumor tissue; this

permits nanomaterials to penetrate and accumulate there (27). Active targeting of nanomaterials is achieved by functionalization of their surface (e.g., by safe-by-design approaches) (28) with bioactive 
molecules such as transferrin, engineered antibodies, enzymes and folic acid, which recognize and interact with cancer-specific targets overexpressed on the surface of malignant cells (22). While efforts to design innovative strategies for active tumor targeting are on-going, there is much debate as to whether active targeting is needed to achieve effective nanomedicine accumulation in tumor tissue (29). For active targeting, the most commonly used targeting moieties are monoclonal antibodies or antibody fragments, antigen binding fragments, and single chain variable fragments (30); the latter being preferable due to their reduced immunogenicity and high target specificity. For example, affisomes are thermosensitive liposomes expressing engineered antibody mimetics of HER2, a well-known cancer biomarker, and have been used to successfully deliver paclitaxel to HER2-positive cancers. In addition, tumor cells have been effectively targeted by coupling tumor-homing peptides to nanoparticles, such as the CREKA peptide that recognizes clotted plasma proteins in tumor vessels and tumor stroma, and the cell-penetrating peptide Lyp-1 (31). We will further describe this nanoproduct in the following sections.

\section{Physico-chemical properties}

Knowledge and control of a nanomaterial's physico-chemical properties are keys to producing an effective nanomedicine, as these properties strongly influence all interactions between the nanomaterial and the various physiological environments that it will encounter during treatment (see Figure 3); therefore, they are fundamental determinants of a nanomedicine's efficacy and toxicity. Nanoparticle size is an important factor for tumor-tissue penetration, as smaller particles can be better transported throughout the tumor. However, small particles also tend to extravasate into the surrounding normal tissue, leading to decreased specificity. On the other hand, larger nanoparticles are more selective as they tend not to extravasate, but their distribution within the tumor can be excessively heterogeneous. This is due to the highly dense extracellular matrix surrounding the tumor, which hinders diffusion of large particles, and to the fact that the aforementioned leakiness of the tumor vasculature is far from homogeneous. The main consequence is an uneven EPR effect, leading to uneven nanomedicine accumulation in the tumor $(27,32)$. 


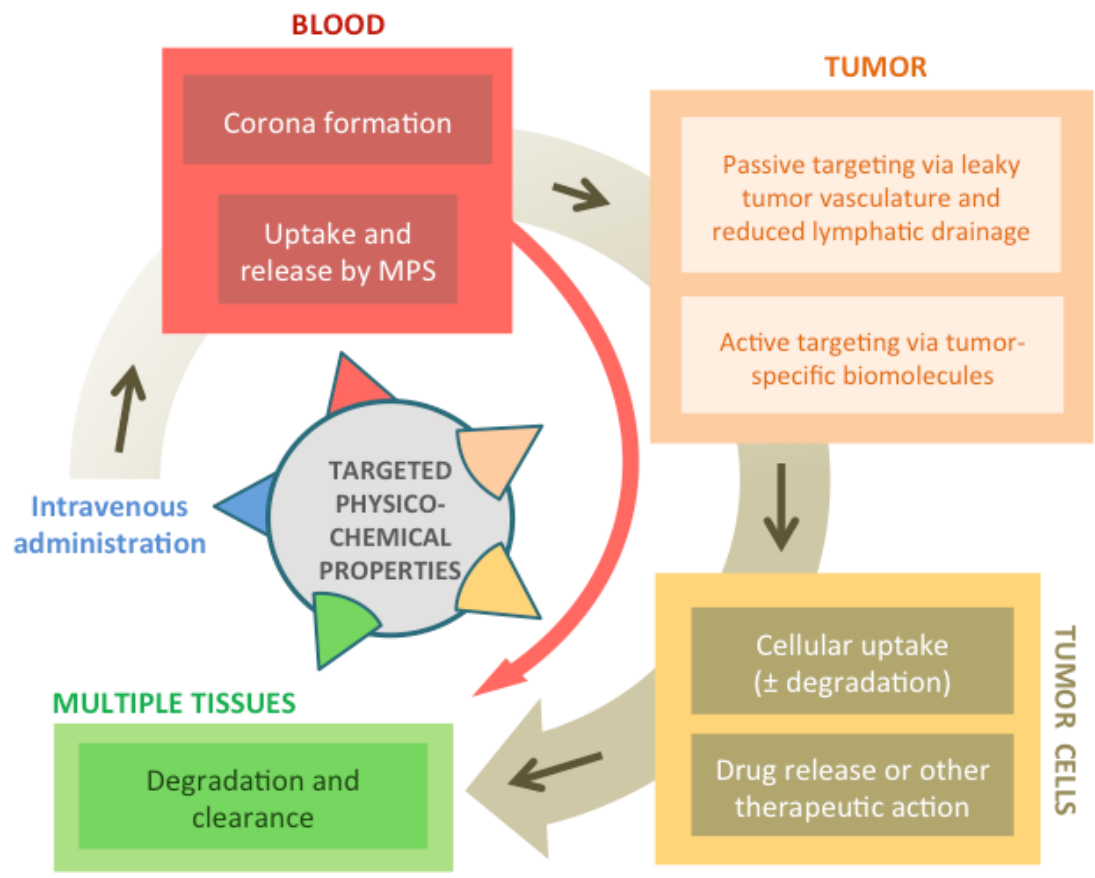

Figure 3. Schematic representation of a typical cancer nanomedicine's journey through the body, highlighting the importance of its targeted physico-chemical properties. These properties strongly influence the transport, distribution, efficacy, toxicity, and persistence of the nanomedicine in the patient. Nanomedicine design requires understanding of the interactions between the nanomaterial and all the different physiological compartments it will encounter during and after administration.

A comparison of the accumulation and effectiveness of different sizes of long-circulating, drug-loaded polymeric micelles with diameters of $30,50,70$, and $100 \mathrm{~nm}$, in both highly and poorly permeable pancreatic tumors, showed that only the $30-\mathrm{nm}$ micelles could penetrate the poorly permeable tumors to a degree sufficient for efficacy. Strategies to enhance penetration and efficacy of larger nanoparticles are available (29), such as the use of angiotensin (33) or transforming growth factor- $\beta$ inhibitor to increase the permeability of the tumor vessels (34), or the control of particles size, aspect ratio and surface charge. Experimental observations have shown that neutral particles can diffuse faster than charged particles, and suggest that optimal particles should initially be positively charged to target the tumor vessels, and 
then switch to neutral after exiting the blood vessels (35). A combination of different strategies is also sought, as shown in one study where researchers found that repairing the abnormal vessels in mammary tumors, by blocking vascular endothelial growth factor receptor-2, improves the delivery of smaller nanoparticles (diameter, $12 \mathrm{~nm}$ ) while hindering that of larger nanoparticles (diameter, $125 \mathrm{~nm}$ ) (36).

Both particle size and composition are considered key factors in determining the mechanism of cellular internalization. This can occur via passive transport through the membrane (similarly to penetrating peptides) and via phagocytosis, macropinocytosis or calveolin/clathrin-mediated endocytosis. In the case of clathrin-mediated endocytosis, most of the nanomaterial will enter the lysosomes for degradation, whereas nanomaterials internalized via the calveolin-mediated process avoid enzymatic degradation and/or inactivation. Ligands such as folate, cholesterol and albumin have demonstrated their ability to promote calveolin-mediated endocytosis. Another physical factor that influences the cellular uptake of nanomaterials is shape, although the extent of this influence remains under investigation (37).

\section{Cellular Internalization of nanomedicines}

The internalization of nanomedicines in the diseased cells of interest is of critical importance because drug targets for various diseases, including cancer, are mainly localized in the subcellular compartments (e.g. DNA) (38). While on one side this need has highlighted the importance of a better understanding of cellular uptake mechanisms in malignant cells, it has also fueled research into the development of more complex nanocarriers capable of targeting not only the malignant cells but also their specific subcellular compartments(39). For this purpose, various strategies have been explored, from endosomal escape to recent approaches used to target the organelles, such as the nucleus, and intracellular cascades. For example, cationic liposomes are generally understood to escape endosomes, which are a compartment of the endocytic membrane transport pathway with slightly acidic $\mathrm{pH}$, by a membrane fusion process where the positive charge of liposomes attracts the anionic phospholipids of the endosomal bilayer, 
resulting in membrane fusion and ejection of the delivered drug molecules into the cell cytosol (40-42). Recently, the release of cationic polymeric nanomedicines from endosomes has been triggered via the "osmolytic" or "proton-sponge effect". According to this strategy, the free amine groups decorating the surface of positively-charged polymeric nanomedicines inhibit the acidification of the endosome local environment as the vesicle matures, thus forcing transmembrane ATPase proton pumps to transport additional protons into the endosome, and inducing osmotic swelling and eventually rupture of the endosome $(43,44)$. Electrostatic repulsion between the charged amine groups would also lead to endosomes swelling and to the release of the nanocarriers by destabilization of the lipid bilayer(45). It should be noted however that, in addition to issues associated with suboptimal endosomal escape, cationic nanomaterials can be associated with significant toxicity and immunogenicity concerns, limiting the pharmaceutical industry interest in their clinical implementation. Regarding the targeting of subcellular compartments, the nucleus is considered to be among one of the most significant targets in cancer nanomedicine. Passive or active nucleus-targeting can be achieved by various strategies(39). The additional complexity of the nuclear membrane that acts as a selective portal for nucleoplasmic transport, however, makes successful localization of nanomedicines in this organelle extremely challenging. Although growing interest and research is focusing on these topics, the concepts of endosome escape and subcellular-targeted nanomedicines are still at their infancy, and more detailed and robust data are needed for their clinical translation.

\section{Protein corona}

Once a nanomaterial enters the human body, it is exposed to environments whose chemical/biological composition is tissue/organ-specific. Depending on the administration route, the nanomedicine will react with different molecules it encounters, thus altering its surface composition and surface properties. One such type of surface alterations is the formation of a layer of proteins adsorbed onto the nanomaterial that continuously exchange with the proteins in the environment. The composition of this "protein 
corona" depends on the properties of the nanomaterial (size, shape, and chemical composition), the nature of the physiological environment (blood, interstitial fluid, lung fluid, cell cytoplasm, etc.) and the duration of exposure (46-51). Interesting recent findings also suggest that the disease state of an individual can impact on the protein corona composition, introducing the idea of a "personalized protein corona" (52).

The protein layer significantly impacts the efficacy and biocompatibility of nanomedicines and is an important factor that drive the success of targeted delivery $(50,53)$, by improving or limiting, the internalization of nanomedicines into tumor cells and/or the drug release. For instance, a recent study showed that stable conjugation of gold nanoparticles with albumin and apoE prior to intravenous administration increases blood circulation time and drives organ targeting, as albumin conjugation significantly increases translocation into brain and targeting of the lungs (54). Similarly, the tissue distribution profile of Abraxane-delivered paclitaxel is influenced by the "protein corona" formation, which can in fact modulate the dissolution rate of the particles and, consequently, the drug release(55). Any change in the composition of the protein corona affects therefore drug distribution and, by extension, Abraxane safety and efficacy. In parallel, the efficacy, toxicity and half-life of the drug released by the nanotherapeutics can also be influenced by its association with plasma/tissue proteins (56). Similarly, to nanomaterials protein corona, the drug interaction with proteins is a dynamic and reversible process where protein-bound drug dissociate into free drug, and vice versa. However, only the free (unbound) form of the drug is available for uptake to cancer cells and can elicit the desired therapeutic effects. The consequence of this is that the ability of a drug molecule to dissociate from proteins determines whether binding to proteins will limit efficacy. Thus, the development of nanodrugs should be based on a good understanding of the "protein corona" and its influence on bioavailability and biodistribution.

Particle degradation and clearance 
The physico-chemical properties of a nanomaterial and the formation of a "protein corona" profoundly impact nanomedicine clearance, and they can either shorten or increase the circulating half-life (30). Clearance is a pharmacokinetic measurement of the rate at which waste substances are cleared from the body, involving both the processes of metabolism in the liver and excretion through the kidneys. A reduction in clearance is associated with an increase of the half-life of the nanomedicine, and consequently with an increase in its bioavailability and efficacy.

One of the principal mechanisms underlying nanoparticle degradation and metabolic clearance is opsonization. This is the process by which a foreign organism or particle gets coated with opsonin proteins, which are present in human blood serum. This makes it more visible to phagocytic cells of the Mononuclear Phagocytic System (MPS), on account of the opsonin receptors in their cell membranes; such cells include monocytes in blood and macrophages in tissue. Macrophages in the spleen and Kupffer cells in the liver can also sequester nanoparticles resulting in accumulation in these organs. Commonly used methods to slow down opsonization of nanocarriers and improve their pharmacokinetic profile include neutralizing the surface charge or adding a hydrophilic coating made, for example, of polyethylene glycol (PEG). However, the hydrophilic coating of nanoparticles may be lost over time, allowing opsonization to occur at later stages.

Interestingly, strategies for drug delivery have also been developed to take advantage of fast uptake by the MPS, as described in the following section. Non-pegylated liposomes (such as Myocet) are taken up very quickly by the MPS, minimizing high plasma concentrations, and are then returned to circulation over time, thus providing gradual, sustained delivery (57).

\section{Design strategies}

Due to the lack of suitable modeling tools, the most relevant design strategies in nanomedicine come from the critical analysis and comparison of the clinical nano-products currently in use for cancer 
treatment in humans. Recently, Dawidczyk and colleagues (57) conducted a case study on six FDAapproved nanomedicines (antibody-drug conjugates, liposomal, and protein drug delivery platforms) that use a variety of strategies to overcome different problems associated with the administration of high doses of the free drug molecules, such as excessive toxicity (Brentuximab vedotin and Trastuzumab emtansine), low solubility (Abraxane), and various side effects (Doxil, DaunoXome, Marqibo). This study suggests that design strategies should take account of the specific constraints imposed by the physiological environments that a nanodrug encounters during its entire journey, from administration to target site to clearance. The authors of the study formulated guidelines for nanodrug design, focusing on stability in circulation; minimization of tissue/peripheral volume; evasion of the MPS; and maximization of tumor accumulation, drug loading, cellular uptake, trafficking and degradation. As is stressed by this study, to maximize nanomedicine targeting, we must improve our understanding of the physiologicallyimposed design constraints that influence biodistribution and drug-release.

\section{Clinically approved nanoparticles for cancer therapy}

Among the various types of nanomaterials developed for nanomedicine applications, liposomes and polymer-based nanoformulations constitute the majority of the nanotherapeutics approved for intravenous administration during cancer treatment (57-59). Liposomes are vesicles with a hydrophilic (polar) cavity surrounded by a bi-layer of amphiphilic phospholipids, whose hydrophobic lipid tails and hydrophilic phosphate heads drive self-assembly in water (60). Polymeric nanoparticles are another major approved platform; these can carry the drug embedded within or conjugated to their surface. Other types of nanoparticle already approved for clinical use are albumin-based particles, micelles and inorganic particles, such as iron oxide nanoparticles for photothermal therapy. The common feature of currently approved nanodrugs for cancer treatment is that they all rely on passive targeting of tumor tissue. 
In this section, we will provide a set of examples of clinically available nanomedicines that are increasing the efficacy of currently available cancer therapies. The most relevant clinically available nanomedicines for cancer treatment are listed in Table 1.

\section{TABLE 1}

\section{Liposomes}

Among the most well-known liposomal formulations is Doxil, which in 1995 became the first nanomedicine to receive FDA approval for treatment of Kaposi's sarcoma; followed by European approval in 1997 under the brand name, Caelyx ${ }^{41}$. In 2013, the use of the generic version, Lipodox, was approved in the US but not for Europe, for the treatment of other tumors, including ovarian cancer (57). Doxil is obtained by encapsulating the anti-cancer drug doxorubicin within PEGylated liposomes, thus increasing the drug circulation half-life and maximizing drug accumulation in tumor tissue. Gabizon and colleagues in 2003 reported a Doxil trial on cancer patients, showing that the ability of the pegylated liposomes to extravasate through the leaky vasculature of tumors, as well as their extended circulation time, results in enhanced delivery of liposomal drug and/or radiotracers to the tumor site. The toxicity profile of Doxil is characterized by dose-limiting mucosal and cutaneous toxicities, mild myelosuppression, decreased cardiotoxicity compared with free doxorubicin and minimal alopecia. The mucocutaneous toxicities are dose-limiting per injection; however, the reduced cardiotoxicity allows a larger cumulative dose than that acceptable for free doxorubicin (61).

Limitations include the fact that liposomes are not generally able to provide the controlled release and stability needed to regulate the kinetic profile and localization of the drug at the tumor site (61-63). Nevertheless, since the development of Doxil more complex structures have been designed starting from the concept of liposomes, to overcome their limitations and allow a longer circulation time, higher drug 
loading efficiency, higher stability and biocompatibility, controlled release properties, and the possibility to delivery multiple drugs $(62,64)$.

Myocet is another nanoformulation designed to deliver doxorubicin to the tumor site within liposomes. It differs from Doxil in that the liposome is not PEGylated, and it is used in clinical practice for treating metastatic breast cancer. The absence of PEG on the surface makes the liposome "visible" to the phagocytic cells of the MPS, therefore decreasing its circulating half-life compared to Doxil, but generating a sustained delivery pattern due to the fact that the MPS cells slowly return the drug into circulation (57). As a consequence, this drug is less toxic and better tolerated than Doxil: for example, it is not associated with palmar plantar erythrodysesthesia, which is the dose-limiting toxicity of Doxil, and shows a significantly reduced incidence of mucositis (65).

DaunoXome is another non-PEGylated liposome, which was developed and approved in the late 1990's. DaunoXome is a formulation of daunorubicin in small unilamellar (single-bilayer) liposomes composed of highly pure distearoylphosphatidylcholine (DSPC) and cholesterol in a 2:1 mole ratio (66). This nanodrug is clinically approved in the USA as a first-line treatment for patients with advanced HIV-associated Kaposi's sarcoma; it is also the subject of a number of clinical trials in leukemia. DaunoXome was originally developed using daunorubicin instead of doxorubicin due to the increased aqueous stability and cytotoxicity of this drug towards certain types of solid tumors. Owing to the small liposome size, its net neutral charge, and the incorporation of both cholesterol and a lipid molecule with a high phase-transition temperature in the liposomal structure, DaunoXome is able to avoid the MPS and has an advantageous circulation half-life of 2-4 hours (65-69).

Depocyt and Margibo are other anti-cancer nanomedicines based on non-PEGylated liposomal nanocarriers. DepoCyt is a sustained release formulation of cytarabine approved in 1999 for the treatment of local intrathecal lymphomatous meningitis, a rare but devastating complication of various neoplastic 
diseases. The liposomal encapsulation is designed to release cytarabine into the cerebrospinal fluid over an extended period of time, increasing the half-life and therefore prolonging tumor exposure, when compared to the non-encapsulated drug. Another benefit of liposomal encapsulation is that it allows for more convenient administration schedules (every two weeks, compared to twice a week for the free drug) $(70,71)$.

In 2012 Marqibo, a vincristine-sulphate-loaded liposome formulation, received accelerated FDA approval for use in adults with advanced, relapsed and refractory Philadelphia chromosome-negative acute lymphoblastic leukemia (ALL) and it is currently being tested for untreated adult ALL, pediatric ALL and untreated aggressive non-Hodgkin's lymphoma $(72,73)$. Marqibo takes the form of a sphingomyelin- and cholesterol-based nanoliposome that encapsulates vincristine; it was designed to overcome the dosing and pharmacokinetic limitations of the conventional pharmaceutical formulations of this drug. The liposomal formulation enables an increase in the drug circulation time, increased target-tissue dose and specificity for optimized drug delivery and allows for dose intensification without increasing toxicity (73). Finally, MEPACT (also called liposomal mifamurtide, L-MTP-PE) is the liposomal formulation of mifamurtide (muramyl tripeptide phosphatidyl-ethanolamine; MTP-PE), a synthetic derivative of muramyl dipeptide, which is used as chemotherapeutic adjuvant. Muramyl dipeptide is a naturally occurring component of bacterial cell walls that acts as an immunotherapeutic agent that potently stimulates macrophages and monocytes to elicit tumoricidal effects (74-76). The encapsulation of MTP-PE into nanoliposomal carriers was developed with the aim of targeting the delivery of the drug to monocytes and macrophages, such as those in the liver, spleen, and lungs $(74,77)$. The liposomal formulation also enhances activation of the tumoricidal capabilities of macrophages/monocytes and extends the drug's biopersistence in the lungs (78). Due to rapid mononuclear phagocytosis of the liposome transporter, LMTP-PE is rapidly cleared from the blood; $0.5 \%$ of L-MTP-PE remains in the plasma after 5 minutes, compared with $93 \%$ when administrated as the free form (79). Due to such rapid clearance, in rabbits and 
dogs, L-MTP-PE exhibits a 10-fold reduction in the level of adverse events, when compared to free MTPPE (74). Liposomal mifamurtide is approved in the $27 \mathrm{EU}-$ member states, as well as Iceland, Liechtenstein, and Norway, for treatment of high-grade, resectable, non-metastatic osteosarcoma in combination with postoperative multi-agent chemotherapy in children, adolescents, and young adults who have undergone macroscopically complete surgical resection. In 2007, the data from a phase 3 trial was presented to the FDA, but the request for approval was denied because the FDA felt that another phase-3 clinical trial, requiring approximately 900 patients with this rare disease, was needed. The drug is currently being tested in several clinical trials in the United States(80).

\section{Albumin-based nanoparticles}

Abraxane is a co-condensate of albumin and paclitaxel, which received FDA approval for clinical use in 2005. These nanoparticles are manufactured by Abraxis Oncology and have been shown to improve the bioavailability of the clinically approved chemotherapeutic agent, paclitaxel. Paclitaxel is poorly soluble as a free drug and, when administered to cancer patients, can result in cardiotoxicity and hypertension. To increase the drug's solubility, albumin-paclitaxel nanoparticles have been developed to encapsulate paclitaxel. Abraxane can enhance drug delivery by passive targeting of the tumor tissue, and shows reduced cardiotoxicity as compared to the free drug (81). When bound to a protein or polymer, paclitaxel is inactive, potentially sparing normal tissue from high-concentration drug exposure and the associated toxicity.

More recently, Abraxane has been conjugated to Lyp-1 and CREKA, with the aim of actively targeting cancer cells. CREKA is a tumor-homing peptide that recognizes clotted plasma proteins in tumor vessels and tumor stroma, while Lyp-1 is a cell-penetrating peptide. Lyp-1- and CREKA-conjugated abraxane has been tested in mice with MDA-MB-435 human cancer xenografts. Lyp-1-abraxane showed enhanced delivery of the drug to the tumor and improved the efficacy of tumor growth inhibition compared to non- 
targeted abraxane; these effects were credited to the ability of Lyp-1 to extravasate and penetrate tumor cells. Unlike LyP-1, CREKA-targeting did not produce the expected result. Although the accumulation of CREKA-abraxane in tumor blood vessels was impressive, distribution within the tumor was similar to unmodified abraxane. Nevertheless, CREKA is an interesting targeting moiety due to its ability to target proteins in clotted plasma leading to the reduction of blood flow, blockage of tumor vasculature and ultimately to tumor necrosis and regression (31).

\section{Polymeric Nanoparticles}

Oncaspar is a PEGylated form of asparaginase (ASNase), a naturally occurring enzyme produced by microorganisms, which catalyzes the hydrolysis of asparagine to aspartic acid. ASNase was identified as a potential chemotherapeutic agent for acute lymphoblastic leukemia (ALL) in 1961 and the potential of ASNase as a childhood-ALL therapeutic treatment was confirmed by a subsequent series of clinical trials (82). PEG conjugation to ASNase shields antigenic regions of the enzyme from immune detection and increases its hydrodynamic radius, resulting in prolonged circulation and retention via decreased metabolic and renal clearance (83). Oncaspar was first approved by the FDA in 1994 for use in ALL patients who developed hypersensitivity to the native form of asparaginase. In 2006, it gained FDA approval as a first-line treatment for ALL, as part of a multi-agent thermotherapy regimen. Owing to its longer half-life, it often takes fewer administrations of Oncaspar to achieve a therapeutic effect similar to that of the native enzyme (84).

Zinostatin Stimalamer was approved in Japan in 1994 for the treatment of primary unresectable hepatocellular carcinoma. It is the conjugation of an antitumor protein neocarzinostatin (NCS) with styrene-malic acid (SMA). NCS exhibits highly potent antitumor activity, but this is coupled with severe toxicity, primarily bone marrow suppression. In addition, its very short half-life of about 1.9 minutes means that maintaining an effective plasma concentration and minimizing systemic toxicity necessitates 
a meticulously controlled infusion velocity based on compartmental pharmacokinetic models. The conjugation of NCS with styrene-malic acid (SMA) increases the drug's half-life and efficacy, while markedly decreasing its toxicity (69).

Finally, nanodrugs can be designed as adjuvant therapies, as in the case of Neulasta (pegfilgrastim), a conjugate form of filgastrim with monomethoxypolyethylene glycol. It has been approved as an adjunct therapy in patients with non-myeloid malignancies receiving myelosuppressive anticancer drugs. In 1991, Filgrastim ( $r$-metHuG-CSF) became the first recombinant human granulocyte-colony stimulating factor (GCSF) to be introduced into clinical practice. Since then, recombinant G-CSF products have emerged as effective adjunct therapies for reducing the incidence and duration of chemotherapy-induced infections due to neutropenia, by stimulating neutrophil proliferation and differentiation in patients with cancer(85): However, filgrastim and other similar G-CSFs require daily subcutaneous injections throughout the chemotherapy cycle. Attachment of a PEG molecule (PEGylation) to filgrastim to create PEG-filgrastim decreases plasma clearance and extends the drug's half-life in the body, allowing for less frequent dosing. Data from randomized controlled studies demonstrate similar efficacy in patients treated once-per-cycle with PEG-filgrastim or twice-daily with filgrastim(86). As Neulasta patent has expired, and given the large economic market of G-CSF therapies in reducing the complications associated with chemotherapy, there has been a large effort in biosimilar development. To date, several filgrastim biosimilars have received US Food and Drug Administration and/or EMA approval and have entered the marketplace(87).

\section{Micelles}

Micelles are colloidal particles ranging in size from 5 to $100 \mathrm{~nm}$. They are similar to liposomes, consisting of amphiphilic molecules that self-assemble to form nanocapsules in water; however, their membranes have a monolayer structure, making the surface of their interior cavities non-polar and therefore suitable 
for hydrophobic cargo. Currently several micellar formulations for anticancer therapy are under clinical evaluation, but only Genexol-PM has been FDA approved (for breast cancer). Genexol-PM is a copolymer micellar nanoparticle that delivers paclitaxel (88).

Inorganic Nanoparticles

NanoTherm is a therapeutic formulation that has received marketing approval in Europe for glioblastoma treatment and is currently under clinical investigation for other solid malignancies. NanoTherm magnetic fluid is an aqueous colloidal dispersion of iron-oxide nanoparticles used to enhance the effect of chemotherapy. Briefly, NanoTherm is injected directly into the tumor site. An alternating magnetic field is then applied and this causes the nanoparticles to rotate rapidly, thereby heating the tumor via the friction generated. Magnetic resonance imaging (MRI) and computerized tomography (CT) can be used to monitor particle accumulation and guide treatment. This novel therapeutic strategy, known as photothermal therapy, has been shown to make cancer cells more susceptible to chemotherapy $(89,90)$.

\section{Nanoparticles under clinical evaluation for cancer therapy}

A number of cancer nanodrugs are currently in clinical trials, demonstrating both the strong potential of this field and the emerging interest in it. In this section, we describe a few examples of nanomaterials under clinical evaluation for cancer treatment, while a more extensive, though not comprehensive, list is provided in Table 2. Unless specified otherwise, information on clinical-trial phase and status comes from the U.S. National Institutes of Health Clinical Trials website (http://clinicaltrials.gov/ct2/home). 


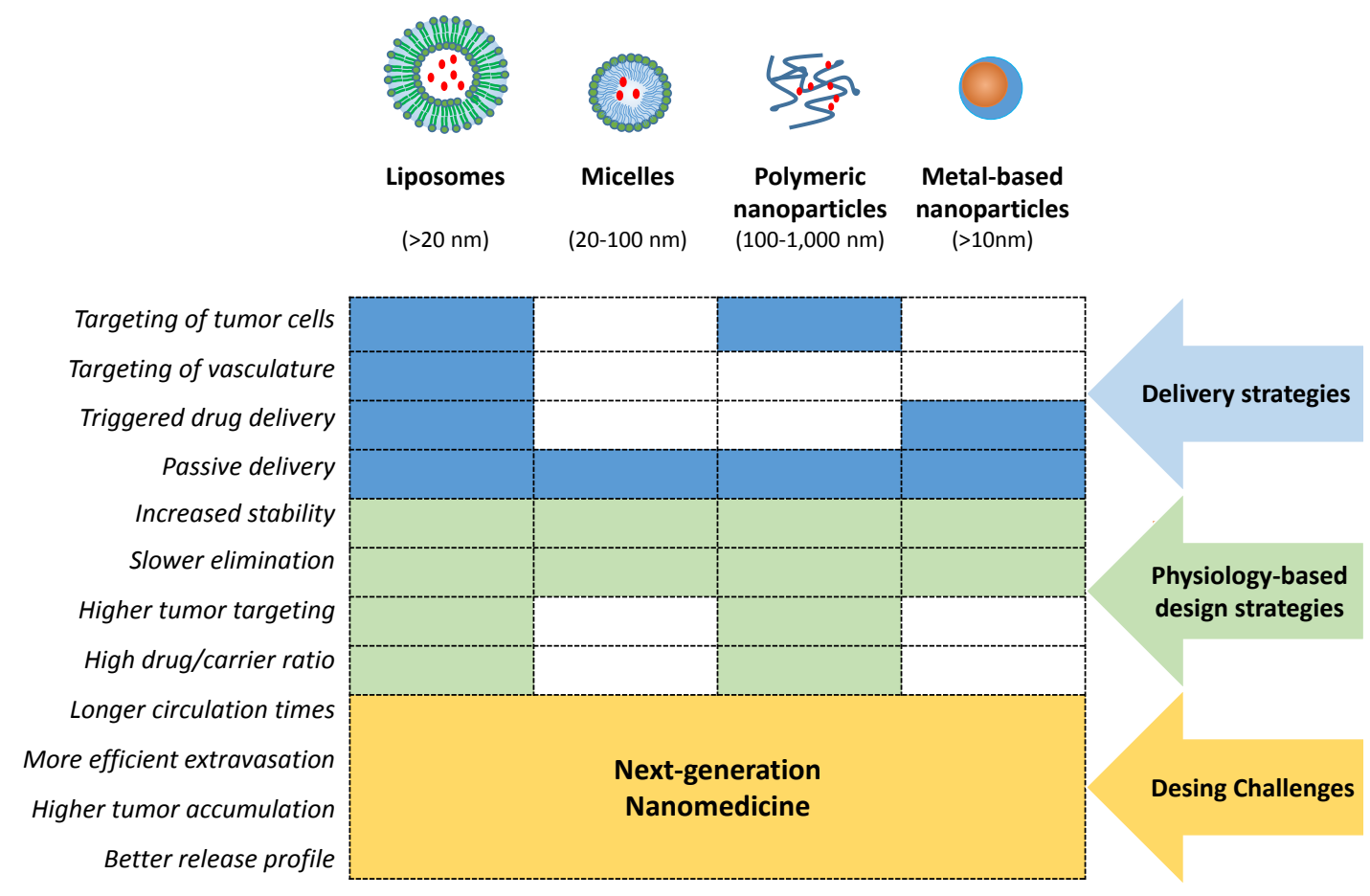

Figure 4. Delivery strategies, physiology-based design strategies and next-generation challenges for different cancer nanomedicine platforms. Liposomes are being developed for all delivery strategies (passive and active targeting of tumor or endothelial cells, and triggered drug delivery). Micelles are mainly being developed for passive delivery of drugs, while polymeric nanoparticles are being used for passive and active delivery. Metal based nanoparticles are mainly being developed for passive delivery and triggered drug delivery (thermaltherapy). All platforms enhance blood stability and longer elimination time compared to coventional drugs, whereas intratumor accumulation and drug/carrier ratio are superior for liposomes and polymeric nanoparticles. Orange boxes highlight the design challenges facing designers of the next-generation of cancer nanomedicines.

We have chosen not to classify the nanomedicines based on the physico-chemical properties of the nanomaterial as in the previous section, but on their tumor targeting strategy. This is due to the fact that, while so far all approved nanodrugs that have been used clinically reach and accumulate in the tumor 
tissue via "passive targeting" mechanisms or in situ administration (e.g., NanoTherm), many nanomedicines currently in development use different targeting strategies (Figure 4). In general, we can classify them into three main categories: tumor-vasculature targeting, passive targeting (among which are triggered drug-delivery systems), and active targeting.

TABLE 2

\section{Tumor-vasculature targeting}

A very promising strategy for cancer treatment is to use nanocarriers to deliver drugs to the endothelial cells of the tumor vasculature. This is achieved by encapsulating the anticancer drug into positivelycharged liposomes that interact with the negatively charged surface of newly developing endothelial cells (which are especially important for the growth of tumor blood vessels), or by designing nanosystems that are functionalized $a d$-hoc with peptides that specifically bind to the surface of activated (i.e., dividing) endothelial cells. Endothelial-cell-targeted nanomedicines possess significant potential for improving the efficacy of anticancer drugs for two main reasons. First of all, damage to endothelial cells will deprive the tumor of the oxygen and nutrients provided by angiogenesis (formation of new blood vessels). The nanomedicines achieve this via a targeted attack on the activated endothelial cells as they divide, leaving non-activated endothelial cells in healthy tissue largely unaffected. This results in specific inhibition of the angiogenesis promoted by cancer-cell secretions and leads to reduced tumor growth. Second, nanomedicines can be engineered to release their contents within the tumor vasculature upon binding to tumor blood vessels, thereby enabling low-molecular-weight drugs to penetrate deeply into the tumor interstitium (91). For these reasons, a significant number of studies regarding nanomedical approaches to the targeting of tumor blood vessels have been published (92-98). In addition, an increasing number of endothelial-cell-targeting nanomedicines are entering and progressing through clinical trials (91). 
For example, Aurimune (CYT 6091) is a product consisting of PEGylated gold nanoparticles that safely improve the delivery of tumor necrosis factor-alpha (TNF- $\alpha$ ), a potent vascular disruptive agent, to tumors. Due to its small size $(27 \mathrm{~nm})$ and PEGylation, Aurimune nanoparticles travel safely in the blood stream to the diseased tissue, avoiding immune cell detection on the way. Concurrently, the local high blood pressure in the tumor forces the nanoparticles to accumulate into the malignant tissue, where TNF- $\alpha$ binds the endothelial cells forming the tumor vasculature. As a consequence, the blood pressure within the tumor vasculature is lowered, allowing subsequent therapies to penetrate the tumor more homogeneously. Studies are under way to allow direct binding of the chemotherapeutic agent to the nanoparticles (99). Aurimune has completed a phase-1 clinical trial, providing encouraging data on its safety and efficient accumulation at the tumor site (100); a phase-2 trial is being prepared (101).

EndoTAG ${ }^{\mathrm{TM}}-1$ is a cationic liposome formulation of lipid-embedded paclitaxel, and therefore targets delivery of the active drug to negatively charged, activated tumor endothelial cells (102). In animal models, cationic liposomes were found to improve drug delivery by targeting the tumor vessels (103), and preclinical studies have shown that such a formulation improves the drug's pharmacodynamics, and maintains its anti-tumoral activity. EndoTAG-1 has been associated with fever and chills, although a tolerable toxicity profile was reported in phase-2 trials (104-106). A controlled phase-2 clinical trial for pancreatic cancer showed significantly increased survival rates for those patients treated with a combination of EndoTAG-1 and gemcitabine. A phase-2 trial in triple-negative breast cancer patients also demonstrated a positive efficacy trend for EndoTAG-1 combination therapy. European and US authorities have granted orphan drug status to EndoTAG-1 in the treatment of pancreatic cancer (107).

Passive drug delivery to cancer cells

Based on a similar chemical approach to the previously discussed and clinically approved drug Abraxane, (also known as Opaxio or Xyotax or paclitaxel poliglumex [CT-2103]) has been developed and tested by 
several groups as a conjugate compound of paclitaxel and $\alpha$-poly-L-glutamic acid. The microtubule inhibitor and radiosensitizer, paclitaxel, is inactive when bound to the polymer, but once inside the tumor cell, enzymes metabolize the protein polymer, releasing active paclitaxel. Abraxane clinical application has been extensively studied for Non-Small-Cell Lung Cancer (NSCLC) in clinical trials up to phase 3 (108), and research has also been conducted into other cancer types, such as breast cancer, recurrent or persistent ovarian cancer, and primary peritoneal cancer (106)'(109). A phase-2 trial of 18 patients with HER2-negative metastatic breast cancer was suspended, as rates of neurotoxicity and late hypersensitivity reactions (HSRs) to the treatment were much higher than expected. HSRs in this case were most likely to occur in later cycles of treatment, suggesting a true drug allergy which is different from the hypersensitivity reactions typically seen with standard paclitaxel (110). However, the safety profile of this treatment has been widely studied by other groups and has been found to have an acceptable tolerability profile (111).

Some liposomal formulations are also moving into the advanced stages of clinical trials (Table 2). Not all trials of liposomal delivery systems produced the expected results in terms of efficacy; nevertheless, they are extremely useful for the subsequent implementation of this technology. One such case is SPI-77, a PEGylated liposomal formulation of cisplatin. In a Lewis lung tumor model, SPI-77 achieved a 28-fold higher tumor AUC than cisplatin, while a 4-fold reduction of cisplatin delivered to kidneys (112). Moreover, SPI-77 exhibited the equivalent anti-tumor efficacy at only half the dose of cisplatin. However, in a following Phase 1-2 study, SPI-077 did not show appreciable efficacy for patients with inoperable head and neck cancer (113). SPI-77 that has also undergone a Phase-2 trial in patients with recurrent epithelial ovarian cancer, but, enrolment in the trial had to be terminated after the first five patients were treated (114). Although four showed stable disease as the best response, and no serious adverse events were recorded, the authors of the study were concerned about the large lipid load and the persistent and prolonged residual platinum in the body. It is worth noting that despite the large cumulative doses 
administered in the tested treatment plan, SPI-77 did not demonstrate any of the platinum-associated dose-limiting toxicities (such as debilitating neuropathy, renal toxicity, and neutropenia) that occur after administration of free cisplatin (115). Other clinical trials with SPI-77 also demonstrated minimal toxicity and a lack of clinical response in NSCLC (116-119). SPI-77 has also been studied in combination with other chemotherapeutic agents and external beam radiation $(120,121)$. While the toxicity profile in these studies was also found to be favorable, the drug did not seem to enhance the efficacy of the treatment regimen. In conclusion, about SPI-77, the clinical trials demonstrated greater safety margin with the liposomal-cisplatin and lack of toxicities typical of the free drug. This case highlights the concept that the challenge is not only to have the payload delivered to the tumor in a relatively safe way (such as, inactive form of platinum), but also to subsequently achieve good release and activation (122).

PEG-based block copolymers are also used as components of various passive-targeting micelles, which are now entering early clinical trials for the treatment of a variety of cancers. For example, SP1049C is a Pglycoprotein micellar formulation of doxorubicin, consisting of doxorubicin and two non-ionic block copolymers (of the Pluronic family). SP1049C has undergone a phase-2 trial in patients with advanced adenocarcinoma of the esophagus and gastroesophageal junction, showing notable single-agent activity, as well as an acceptable safety profile with neutropenia as the principal toxic effect (123). Another example is NK911, a polymeric micelle system, comprising PEG and poly(aspartic acid) and designed for the enhanced delivery of doxorubicin. NK911 completed a phase-1 clinical trial phase in 2004 (124). NC6004, a PEG-poly(glutamic acid) block copolymer micelle encapsulating cisplatin, is an another example of a conjugated polymer-drug nanomedicine in late-stage clinical trials for several types of cancer (125). Paclical is a micellar formulation of paclitaxel that has recently received orphan drug designation by the FDA, and is currently in Phase-3 trials for ovarian carcinoma (80). Finally, CRLX101 is a camptothecincyclodextrin conjugate that has demonstrated improved pharmacokinetics in both preclinical and clinical studies (126). 


\section{Passive targeting with triggered drug delivery}

One highly promising class of anticancer nanomedicines currently being assessed in clinical trials is based on systems that release their bioactive content or otherwise exert their pharmacological effect upon exposure to external stimuli, such as heat, light, ultrasound, or magnetic fields (91). Such stimuliresponsive nanomedicines hold significant clinical potential, since they are designed to release the conjugated or entrapped chemotherapeutic or adjuvant drug only in response to locally confined stimuli, thereby maximizing drug release in the pathological tissue while preventing damage to healthy tissues. Triggered drug delivery systems developed so far are either injected locally into the tumor tissue or exploit the EPR effect to reach the malignant target.

Some of the micelle-based drug delivery systems currently undergoing clinical trials can be functionalized with $\mathrm{pH}-$, thermo-, ultrasound-, or light-sensitive block copolymers to allow for controlled micelle dissociation and triggered drug release (88). Thermosensitive block polymers can also be introduced into liposomes bearing an anti-cancer drug and an imaging agent, thus making the drug-delivery system both temperature-triggered and multifunctional (127).

The downside of triggered drug-delivery systems is the difficulty of tuning the stimulated drug release. The main consequence is that these systems often release significant amounts of drug without actually being triggered, or they are so stable that the triggering conditions required to induce drug release are too severe and may become toxic. To overcome these issues, efforts are currently being undertaken on two sides: (1) to improve the stimulus responsiveness and (2) to develop more suitable hardware tools that are capable of administering external stimuli more effectively and more selectively to the target tissue (89). Some representative examples of these attempts are discussed in more detail below.

ThermoDox is a thermosensitive liposome that delivers doxorubicin exclusively when an external heat source is applied, and its approval for clinical practice is expected soon. ThermoDox has shown particularly 
unique and effective therapeutic potential by limiting its anti-cancer activity until the target tissue is reached, thus reducing the toxic effects $(69,128,129)$. The thermosensitivity of ThermoDox liposomes is associated with its chemical structure; it is composed of dipalmitoylphosphatidylcholine (DPPC), monostearoylphosphatidylcholine (MSPC), and DSPE-MPEG-2000. DPPC, which has a gel-to-liquid crystalline phase transition temperature (Tc) of $41.5^{\circ} \mathrm{C}$, induces membrane instability in the ThermoDox liposome at temperatures around its Tc. The liposome has a diameter of about $100 \mathrm{~nm}$, so it is rapidly identified by the MPS and concentrated in the liver, thus making it an ideal candidate for the treatment of hepatocellular carcinoma (HCC) (128). In order to deliver the thermal energy required to release the encapsulated doxorubicin, RadioFrequency Ablation (RFA) is chosen as it is already widely used in the treatment of $\mathrm{HCC}(69)$. TherermoDox is currently undergoing a phase-3 clinical trial in conjunction with RFA for the treatment of HCC, a phase-1/2 clinical trial with approved hyperthermia for the treatment of breast cancer recurrence at the chest wall, and it has completed a phase- 1 study for treatment of liver metastasis.

Paramagnetic nanomedicines have also attracted much attention as promising anti-cancer therapeutics and several forms of electromagnetically activated NPs are currently nearing or progressing through clinical development. For instance, NanoTherm, which has already obtained clinical approval for the treatment of glioblastoma, is undergoing clinical trials for further tumor types $(3,130,131)$. AuroLase is another example of this class of nanomedicine. AuroLase nanoparticles have silica-based cores and gold outer shells (Auroshell). Once administrated intravenously to the patient, a fiber-optic probe is used to deliver infrared laser radiation to the tumor site, inducing localized heating $(132,133)$. AuroLase is currently under investigation in a phase- 1 trial for the treatment of advanced lung cancers, as well as advanced head and neck tumors. NanoXray which is being developed by Nanobiotix, uses paramagnetic, electron-dense hafnium-oxide nanoparticles that accumulate within the tumor. Here, they enhance the effect of radiotherapy by absorbing X-rays, leading to ejection of electrons from the nanoparticle and, 
thereby, formation of damaging free radicals within the tumor cells (134). Since this treatment effectively increases the dose of radiation at the tumor site without significantly impacting that experienced by the surrounding healthy tissue, it can produce similar efficacy at lower doses; thus, reducing adverse effects. The NanoXray ${ }^{\mathrm{TM}}$ technology includes three similar nanoparticle products that are optimized for three different routes of administration; the product designed for intratumoral injection, NBTXR3, is currently at phase- 1 of clinical trials for the treatment of soft-tissue sarcoma and locally advanced squamous-cell carcinoma of the oral cavity and oropharynx.

\section{Active drug delivery to tumor cells}

While the potential benefit of a nanomedicine that uses an active targeting mechanism is widely accepted, the translation of such technologies into clinical practice has been slower than expected. The first examples of cell-specific targeting using ligand-conjugated liposomes were in fact described in 1980, and since then, a great number of targeted nanoparticles have been developed for drug delivery and imaging. Nevertheless, only a small fraction of these have progressed into clinical trials $(62,135)$.

Transferrin and folate are two of the most commonly used bioactive ligands for actively targeting nanomedicines to tumor cells $(136,137)$. For example, MBP-426 is a liposomal formulation functionalized with transferrin, and it has recently completed a phase-1 clinical trial in patients with advanced or metastatic solid tumors (80). Transferrin has also been employed as a targeting ligand for the cyclodextrinbased nano-drug candidate CALAA-01. The active ingredient in CALAA-01 is a small interfering RNA (siRNA), which inhibits tumor growth by inducing degradation of mRNA for the M2 subunit of ribonucleotide reductase (R2). CALAA-01 is designed for treatment of various solid tumors, and in 2008, it became the first targeted siRNA nanomedicine to be administered to humans, demonstrating that systemic administration of siRNA can produce inhibition of a specific gene in humans (138). Between 2008 and 2010, nineteen patients were enrolled in the phase 1a clinical trial, but 2 patients experienced dose 
limiting toxic (DLT) effects. In 2012, a phase-1b clinical trial of CALAA-01 for treatment of solid tumors was started, but it was soon terminated because, two out of five patients experienced dose limiting toxic effects (139).

A very promising nanodrug candidate, currently in phase- 2 clinical trials for NSCLC and prostate cancer, is BIND-014, a polymeric nanoparticle that delivers docetaxel by actively targeting the prostate-specific membrane antigen (PSMA), a clinically validated tumor antigen expressed on prostate-cancer cells and in the vasculature of most non-prostate solid tumors. BIND-014 is manufactured via innovative self-assembly techniques using pre-functionalized polymers, and tumor targeting is achieved through an RNA aptamer (Apt), which binds specifically to PSMA on the surface of cancer cells. Poly(D,L-lactide-co-glycolide) (PLGA) is the basic component of the nanocarrier that allows for controlled drug release, and it is conjugated with polyethylene glycol (PEG) to increase circulation half-life (140).

\section{Challenges limiting clinical translation of cancer nanotherapeutics}

Despite the huge investments and the astonishing number of scientific publications regarding "nanotechnology for cancer treatment" in the last 10 years (Figure 5), the translation of oncological nanomedicines into clinical practice has been slow compared to that for small-molecule drugs $(141,142)$. In fact, the majority of nanomaterials designed for clinical use are barely at the stage of in vivo evaluation, and even fewer have reached clinical trials. On the scientific and technological side, existing knowledge gaps are delaying the development of widely accepted modeling and predictive screening strategies, which would speed up the process between conception of innovative nanomedicine platforms and clinical approval. Challenges also lie in integrating the expert input required from a wide variety of disciplines. Nanomedicine requires a greater-than-ever effort to integrate all the relevant disciplines, to go beyond the limits of discipline-specific knowledge and jargon, and to connect the practical needs of medicine to the enormous potential of nanotechnology. In addition, clear regulatory guidelines for the translation of 
nanotechnology-based therapies into clinically marketable products are lacking; this is discussed in more detail below.
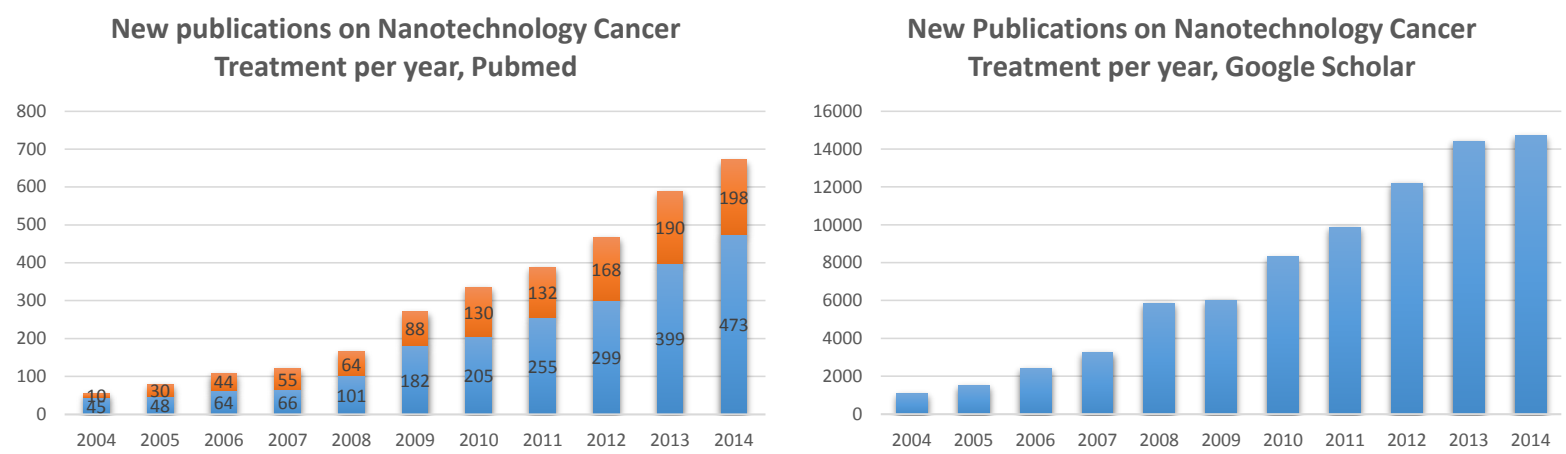

Fig 5: Publication trend on the topic of "nanotechnology for treatment of cancer". Two public databases were searched for "nanotechnology AND cancer AND treatment", sorted by year of publication from 2004 to 2013. A: Pubmed medical sciences database (http://www.ncbi.nlm.nih.gov/pubmed) displayed a total of about 2,500 papers, with about 800 of those being reviews (orange bars) and the remaining majority being original-research papers (blue bars). B: Google scholar (scholar.google.com) searches many scientific databases and provides a more extensive list of publications. With the exclusion of patents and citations, it displayed a total of almost 65,000 publications within the same period.

Scientific and technological challenges

Although one of the main advantages of nanomedicines is to enable the targeting of drugs to diseased organs, controlling and predicting their distribution in the human body requires further investigation (27).

The structure of the tumor microenvironment and vasculature is abnormal, with a dense extracellular matrix surrounding the cancer cells, a particularly uneven blood flow and highly heterogeneous vessel permeability. While on the one hand, the EPR effect can positively influence the passive targeting of tumor 
tissue by nanomedicines, these structural factors can also negatively impact the delivery, penetration, and homogeneous distribution of nanomedicines in tumors $(27,140)$; posing a problem for both passive and active targeting strategies. Furthermore, understanding of the relationship between nanoparticle structure and behavior in biologically complex environments like the human body is far from complete, making it difficult to predict nanocarrier biodistribution and to develop broadly applicable nano-specific modeling tools (currently, none are available). These limitations make rational design of nanomedicines difficult and mean that a large number of in vitro and in vivo (animal) screening approaches are necessary.

Nanomedicine safety is considered, as for standard pharmaceutical drugs, on the basis of the risk-benefit ratio, making it a broader question than just the toxicity of the nanomaterial. Clinical translation is suffering from the lack of widely accepted in vitro screening platforms that can rapidly evaluate both the efficacy and toxicity of nanomaterials in a manner that produces good correlations with in vivo outcomes (143-146). For example, there is a need for high-throughput methods for evaluating the binding and internalization of nanomaterials by cells, and the interaction of nanomaterials with plasma proteins and the complement system. Biomarker assays and other testing methods that are classically applied for small molecular drugs have often proven unsuitable for nanomedicine candidates, as their nano-specific properties frequently interfere with test readouts (147). In addition, classical molecular endpoints may lose their predictive meaning when it comes to testing nanomedicines. Even the type of cell-culture model to be employed remains a matter of debate: most in vitro toxicity studies are carried out using cells cultured in monolayers (two-dimensional); however, since the penetration of nanomaterials into cells or tissues is influenced by interactions between its own properties (e.g., charge, shape, functionality, and material composition) and those of the three-dimensional (3D) tumor microenvironment, novel 3D cell systems may be required. Nanomaterial sedimentation during in vitro testing is another crucial factor that needs to be considered when choosing the cell-culture model. Sedimentation can significantly affect the rate and extent of nanodrug uptake by cells (148), and thus ultimately influence their 
pharmacological/toxicological response (149). 3D tumor spheroids have been recently proposed as the "standard-to-be" for development and optimization of new chemotherapeutic agents $(150,151)$. Such spheroids can in fact closely mimic the complex tissue organization of a tumor, creating valuable models that allow for reduced animal use in testing, thereby representing methodological progress that is consistent with the "3Rs principle" of ethical animal experimentation (Replacement, Refinement, and Reduction). These innovative systems may become particularly useful in vitro testing platforms for assessing the toxicity and efficacy of newly developed nanomedicines, and for accelerating their translation into approved cancer treatments (152).

Another weak link in the nanomedicine translation pipeline is the physico-chemical characterization step. In order to expedite the transition from benchtop efforts to clinically effective products, investigators need to employ methods that are appropriate, standardized and widely accepted (11). Characterization is at the base of the testing chain, and it enables us to correlate nanomedicine properties to toxicological consequences and biological responses. Nanomaterials are routinely characterized with respect to size, surface charge, and ligand density, but consensus does not exist on the most suitable characterization techniques to be employed. Similarly, nanomedicine characterization is often performed under conditions that do not adequately reflect the complexity of the physiological environment. Analysis is often carried out without considering that, when nanomaterials come in contact with blood, they are subject to several modifications and can be readily coated with a complex "protein corona", dramatically altering their surface characteristics and ultimately their effects in vivo (153). There is therefore a drive to incorporate novel evaluation methods and approaches in the nanomedicines development pipeline; approaches that integrate, for example, microfluidic technologies, the latest of which have emerged as promising tools for creating in vitro microenvironments that mimic in vivo conditions. As it has been recommended by the US National Characterization Laboratory (NCL), the most effective way to determine the physico-chemical properties that influence a nanomedicine's efficacy and toxicity, is to use multiple methods based on 
different principles. According to the $\mathrm{NCL}$, "this type of integrated testing will allow identification of scientific and regulatory gaps in nanomedicine and help developers and regulators strategize new ways to bridge those gaps" (99).

Once a new candidate nanomedicine is ready for clinical evaluation, important technological challenges arise based on the difficulty in reproducibly scaling-up the production process with identical and qualitycontrolled properties, supplied in sufficient quantities for preclinical and clinical applications (154). Problems such as batch-to-batch variation and the unsuitability of classical methods to test for impurities, contaminations and aggregation can severely limit the pace of clinical translation $(142,146,147,154)$. Since the safety and efficacy can be influenced by small variation in the physico-chemical characteristics which can compromise the biological behaviors or pharmacological profiles (154), there is the need for a characterization cascade, from physico-chemical, to in vitro and in vivo testing, which will screen and accelerate the translation of nanomedicine (155). Consequently, the assessment of the long-term in vivo effects of nanomedicines is of fundamental importance, and represents a major scientific challenge. It accumulates the uncertainties from all previous steps in the evaluation process, from physico-chemical characterization to in vitro and in vivo toxicity and efficacy screening, and adds to this its own technical difficulties (156). Despite the lack of suitable predictive pharmacological modeling methods, preclinical studies of nanomedicines must confront the unique aspects of animal-study design, such as (1) difficulties in evaluating biological potency and in selecting analytical methods that can determine the in vivo stability of nanomedicines, and (2) the appropriateness of current dose-scaling techniques for estimation of the first-in-man dose from preclinical data (157). As a result, knowledge obtained from physico-chemical and preclinical testing is often of little value in predicting the clinical outcomes of new nanodrugs (146); this is the most obvious case when the characterization is not performed under biologically relevant conditions. For instance, as stated by Crist et al. within the NanoCharacterization Laboratory (NCL), there are several parameters, including size and charge, which vary with dispersing medium and the 
microenvironment (158). Thus, without proper and adequate physicochemical characterization (PCC) of a nanoparticle and its surface ligation, in vivo toxicity results may be misleading and ultimately meaningless. Furthermore, an accurate quantification of the active pharmaceutical ingredient (API) within the engineered nanomedicine is not always immediate and can lead to miscalculation of the maximum tolerated dose (MTD) in vivo. For instance, in vitro characterization carried out with only buffer solutions is the wrong model to use for testing a nanomedicine especially if this has to be injected intravenously in an immunocompromised patient where the microenvironment is totally different. The size, charge and consequently drug payload will therefore change the pharmacotoxicity profile of the nanomedicine.

As stated above this can be critical in influencing the preclinical and clinical studies outcome $(13,154)$. Furthermore, since the international nanomedicine industry is currently tackling the next generation challenges in order to deliver effective solutions to patients (13), consideration across the whole nanomedicine drug-development process and how to deliver large volumes of high quality nanomedicine is also needed and encouraged across all stakeholders (159). Practical guides, considerations and challenges from the industrial perspective have been presented in the context of improving the supply chain towards an increased translation of nanomedicine drugs into clinical studies and trials $(13,99,154$, 155).

\section{Regulatory challenges}

Scientific and technological issues in nanomedicine need to be addressed in an integrated environment, where the entire stakeholder community can share needs and issues during the early stages of development. Integration is not only needed among the various technical disciplines listed in Figure 1, it is also essential across the broader fields of academia, industry, and regulation. 
The introduction of integrated multi-assay approaches will be key to providing regulators with decisionmaking tools that allow for classification of nanomedicines at the early stages of testing, thereby determining the most appropriate methods for more advanced assessment.

From a regulatory point of view, the lack of a clear and widely accepted definition of "nanomaterial" (12, 99), and thus "nanomedicine" (59), is another source of uncertainty. The FDA initially adopted the 2007 National Nanotechnology Initiative (NNI) definition by which nanotechnology is defined as involving all of the following elements: (1) the research and technology development at the atomic, molecular or macromolecular scale leading to the controlled creation and use of structures, devices and systems with a length scale of approximately 1-100 nm; (2) creating and using structures, devices, and systems, which have novel properties and functions as a result of their small and/or intermediate size; and (3) ability to control or manipulate on the atomic scale. In 2011, the FDA issued a draft guidance for industry entitled "Considering Whether an FDA Regulated Product Involves the Application of Nanotechnology" (160), where it noted that the FDA chose not to adopt a regulatory definition of nanotechnology or related terms. In determining if an FDA-regulated product involves the use of nanotechnology, the FDA parameters are:

(1) whether the engineered material or end product has at least one dimension in the nanoscale range (approximately 1-100 nm); or

(2) whether the engineered material or end product exhibits properties or phenomena, including physical or chemical properties or biological effects, that are attributable to its dimensions, even if these dimensions fall outside the nanoscale range, up to $1 \mu \mathrm{m}$.

From the European perspective, the European Commission (EC), provided an official and more detailed definition of "nanomaterial" in the Recommendation 2011/696/EU. According to the EC Recommendation: 
- Nanomaterial means a natural, incidental or manufactured material containing particles, in an unbound state or as an aggregate or as an agglomerate and where, for $50 \%$ or more of the particles in the number size distribution, one or more external dimensions is in the size range 1$100 \mathrm{~nm}$.

- In specific cases and where warranted by concerns for the environment, health, safety, or competitiveness, the number size distribution threshold of $50 \%$ may be replaced by a threshold between 1 and 50\%.

The definition is recommended for all fields of application of nanotechnology, where a distinct regulatory definition is not provided as in the case of nanomedicine. Yet it is clear that nanomedicines are often not within the 1-100 nm size range, which is critical in nanomaterials where quantum effects are paramount for their use (e.g., in electronic or optical devices), yet this size limitation is not usually relevant from the point of view of drug delivery or efficacy. For example, small liposomes of 30-100 nm in diameter are less stable than their larger counterparts, due to their higher surface curvature and resulting surface tension; therefore, liposomes in a size range of 150-200 $\mathrm{nm}$ have a greater blood residence time. The EPR effect typically operates in the range of $100-400 \mathrm{~nm}$, and phagocytosis of nanoparticles via macrophages can be accomplished in ranges beyond the arbitrary cut-off of $100 \mathrm{~nm}$ (12). With respect to such considerations, the EC acknowledged that an upper limit of $100 \mathrm{~nm}$ is not scientifically justified across the whole range of nanomaterial applications, and noted that "special circumstances prevail in the pharmaceutical sector" by stating that the recommendation should "not prejudice the use of the term "nano" when defining certain pharmaceuticals and medical devices" $(161,162)$.

The European Medicine Association (EMA), the EU agency responsible for scientific evaluation and supervision of medicines, refers to nanotechnology as "the use of tiny structures - less than 1,000 nanometers across - that are designed to have specific properties" on its website. The EMA has also 
provided its own working definition of a nanomedicine: a system designed for clinical applications that is composed of at least one nanoscale component, resulting in specific advantageous characteristics, such as better targeting and bioavailability of therapeutics, new modes of therapeutic action, and nanostructured surfaces/scaffolds for engineered tissues $(66,143)$.

Ultimately, even though the US NNI and EC definitions of nanotechnology and nanomaterial are substantially different, the fundamental difficulty in providing a clear and unequivocal definition of a nanomedicine seems to be shared by both US and EU regulators.

\section{Regulating clinical translation}

According to the EMA, the existing EU regulatory framework can accommodate nanomedicine and its emerging challenges through adaptations (68), while experience and knowledge acquired over the years will allow the need for nanomedicine-specific guidance to be addressed.

Aiming to address the current lack of clear regulation for nanomedicines, the EMA has already recognized the particular challenges that are arising from "nanosimilars" and "next-generation nanomedicines" (143). Nanosimilars are similar versions (generics) of the "originator" nanomedicines, follow-on products, which are developed when first-generation nanodrugs are coming off-patent. As for generic drugs, the equivalence of nanosimilars needs to be demonstrated through a stepwise set of comparison studies that address quality, safety, and efficacy. While this type of comparison is standardized for conventional drugs, on account of their high degree of complexity, special considerations are needed to ensure the equivalent performance of nanomedicines (11). Physico-chemical and pharmacokinetic sameness is not sufficient to indicate that two nanotherapeutics with different formulations will perform comparably in a clinical setting. For example, the Abraxane case suggests that bioequivalence of paclitaxel cannot be demonstrated with pharmacokinetic assessment of free and protein-bound paclitaxel alone. The kinetics of exchange is in fact dependent on the binding substrate and the microconditions offered by the 
nanotherapeutic formulation(163). In order to address such considerations and allow regulators to establish this generic-like "nanosimilar" status, understanding of how the nanomedicine's physicochemical properties influence its distribution and clearance in the human body, again, becomes key (99). Experience with the first nanosimilars has shown that additional clinical studies should be required to ensure the safety and efficacy of a nanosimilar drug, as even nanomedicines that have been safely used for many years are heavily challenged when it comes to follow-on versions (99). To pave the way for nanosimilars, the EMA issued several reflection papers in 2013 with a view to developing guidelines for both nanomedicines and nanosimilars (143); these are listed in Table 3.

\section{TABLE 3}

Additional regulatory consideration is required for so-called "next-generation" nanomedicines, which are multimodal nanomedicines that act as pharmaceuticals, imaging agents, and combination products. The challenges faced in evaluating such complex nanomedicine products are addressed by the EMA in one of its 2013 papers, where they emphasized the need for sensitive and accurate analytical methods to characterize not only the nanomedicine as a whole, but also each of its individual components, and their interactions with the physiological environments encountered during clinical application (143). In the same paper, the agency also highlights the challenges faced in establishing regulatory requirements and assessment standards for preclinical and clinical studies of these products.

Furthermore, the present lack of clear definitions and regulatory guidance may lead to situations where next-generation nanomedicines fall within several categories of regulation; owing to their complex structures and multiple modes of action, be they mechanical, chemical, pharmacological, or immunological. In Europe, a medicinal product whose action is physical or mechanical in nature is governed by European medical devices regulations and evaluated by EU member states, whereas in the case of chemical, pharmacological, or immunological action, the product is considered as a 
pharmaceutical drug and thus evaluated by the EMA. Thus, regulation uncertainty and overlap may result if the manufacturer needs to submit its application to different bodies, or can choose among different approval pathways with different procedures and different risk evaluations and assessments. In addition, according to European regulations on medical devices, which sets common rules for the National Authorization Authorities of EU member states, nanomaterial-based products should be evaluated following the conventional protocols used for macro-sized medical devices, with the only exception being that medical devices containing nanomaterials are automatically ranked as Class III, thus being considered as potentially highly dangerous. Debate about this classification is intense among stakeholders, with some asking for additional requirements specific to nano-based medical devices, which should for example include additional specific risk assessments and biological studies in accordance with the standard procedure for biocompatibility assessment, ISO 10993. In this context, September 2012 saw the European Commission propose a regulation on medical devices with specific rules for this class of platform, and a re-categorization that transfers regulatory oversight from the EMA to member-state authorities (164). So far, the EMA has evaluated 11 marketing-authorization applications for nanomedicines, of which, 8 have been approved and 3 withdrawn, and granted orphan drug status to 10 nanomedicines under development (143). This number is smaller than in some of the larger EU countries such as France, where 36 nanomedicines have been licensed (165). An example of the disparities in the international classification of nanomedicines is given by the aforementioned nanomedicine company, Nanobiotix. In Europe, its technology, NanoXray has been categorized as a medical device, while in the US, the FDA has classified it as drug. As a result, Nanobiotix will probably reach the market more quickly in Europe than in the US because the European national authorities responsible for licensing medical devices require fewer clinical trials than the FDA, when licensing pharmaceutics.

Taking a closer look at situation in the US: according to FDA, regulations can only be based on the current best information and the product has to be fundamentally safe and effective, independent of whether it 
employs a nanomaterial, and evaluation is based on case-by-case assessment. In 2007, the FDA stated that existing regulations were sufficiently comprehensive to ensure the safety of nanoproducts because these products would undergo premarket testing and approval either as new drugs under the New Drug Application (NDA) process or, in the case of medical devices, under the Class-3 Premarket Approval (PMA) process $(12,166)$. This conclusion was based on the assumption that the regulatory requirements in place would detect toxicity following the required safety studies, even if nanoproducts presented unique properties related to their size. Many experts criticized this view, especially since most FDA-approved nanoproducts obtained approval based, in whole or in part, on studies of non-nano versions of the drug, so that the nanoproducts did not undergo the full PMA process or NDA process (12). In 2011, the FDA reopened the dialogue on nanomedicine regulation by publishing proposed guidelines on how the agency will identify whether nanomaterials have been used in FDA-regulated products(167). The FDA's purpose here was to help medical product developers identify when there is a need to consider the regulatory status, safety, effectiveness, or health issues that could arise from the use of nanomaterials or nanotech in FDA-regulated products. In 2012, the FDA commissioner summarized, in general terms, a "broadly inclusive initial approach" with respect to "nano-governance" in a two-page policy paper published in Science. This paper stated that the "FDA does not categorically judge all products containing nanomaterials or otherwise involving the application of nanotechnology as intrinsically benign or harmful. As with other emerging technologies, advances in both basic and applied nanotechnology science may be unpredictable, rapid, and unevenly distributed across product applications and risk management tools. Therefore, the optimal regulatory approach is iterative, adaptive, and flexible" (168). Most experts in the nanomedicine field continue to criticize the FDA effort to regulate nanotechnology, pointing to the fact this delay in addressing nano-specific regulation could have a very harmful effect on investors, public confidence, and commercialization efforts (12).

Harmonization of regulations 
Harmonization among nanomedicine regulations in different countries is highly sought after. In 2009 , the EMA's Committee for Medicinal Products for Human Use (CHMP) established an ad hoc expert-group meeting on nanomedicines. This group of selected experts from academia and the European regulatory network supported the agency's activities by providing specialist input on new scientific knowledge, reviewing the guidelines on nanomedicine development, and extending its regular contacts with nonEuropean agencies on nanomedicines. CHMP chairs schedule regular meetings with the FDA, as well as the licensing authorities of Japan, Canada, and Australia. The main goal of these interactive communications is to guide the development of nanomedicines towards timely and effective clinical translation, and the reflective paper on block-copolymer micelles, written jointly by the agency and Japan's Ministry of Health, Labor, and Welfare (MHLW), is one of the results of these international meetings $(165,169)$.

The dialogue between academia and industry is also critically important in the whole process, as those knowledge gaps that prevent industry from investing more in nanomedicine development can only be tackled by academic research expertise. On the other hand, an increased focus on industry could greatly benefit academic research, especially in an applied-science field like nanomedicine. In early 2009, the European Commission, together with the European Society for Nanomedicine (ETPN), suggested that research and development activities should be focused on identifying translatable trends in research and understanding their expected impact on applications, production, and markets; thus, enabling the finetuning of research funding to target areas with greater potential for large-scale health benefits and commercialization. Successful translation of academic research into industrial and clinical applications starts from the concept of nanomedicine as an applied science, and therefore application must be the driver of research efforts. A number of Initiatives are being pursued that promote the application of nanomedicine by strengthening the links between academia and industry. One of these is the ETPN, which was founded in 2007. It is led by industry and supported by the European Commission, with the aim 
promoting the application of nanotechnology to achieve breakthroughs in healthcare and to intensify innovation in nanobiotechnology (165).

\section{Conclusions}

Nanotechnology is a key enabling technology, whose application in medicine ranges from diagnostics to therapeutics to medical devices. As such, it has the potential to provide major health benefits for all. The medical use of manufactured nanomaterials is currently in its infancy, with some anticancer nanomedicines already on the market, but most nanodrugs are still in progress through the different stages of the pharmaceutical pipeline. As is often the case for emerging technologies, nanomedical innovation is running at a faster pace than the acquisition of data on its safety, while regulation is already struggling to keep up with the advent of "nanosimilars" and "next-generation" nanomedicines.

The majority of cancer nanodrugs on the market are liposomes and polymer-based nanoformulations that lower toxicity and enhance delivery of chemotherapeutics via passive targeting, which is based on the "Enhanced Penetration and Retention (EPR) effect" that stems largely from the leaky vasculature and reduced lymphatic drainage in tumor tissue. Complexity is higher for next-generation cancer nanotherapeutics currently in the development pipeline, in terms of hybrid structures, surface physicochemical characteristics and mechanisms of delivery and action. Complex formulations based on liposomes, micelles, polymeric nanoparticles and metal nanoparticles are in current trials, these aside from the passive targeting delivery through EPR effect, are attempting to reach the tumor through active targeting systems or are activated by an external source of energy only when the malign tissue or cells are specifically reached.

Technical issues are, to some extent, limiting the clinical translation of nanomedicines. Future developments that could greatly improve the speed and efficiency of translation include: 
- standardized and validated in vitro assays for testing of nanomedicine safety and efficacy;

- in vitro/ex vivo models relevant to the specific routes of administration used by nanomedicines;

- improved understanding of the absorption, distribution, metabolism, and excretion (ADME) of nanomedicines in vivo, and their relationship to critical product characteristics;

- development of in silico modelling approaches to predict the biological and toxicological effects of nanomedicines in the human body;

- broader understanding of factors that define interactions between nanomaterials and living systems, such as surface binding of biomolecules in vivo (protein corona) $(144,165)$.

Yet the most direct challenge to translation of this research is the lack of precisely defined regulatory requirements, as this translates into uncertainty for investors and reduced public acceptance. In addition, knowledge gaps and unclear scientific data concerning nanomedicine characterization, biopersistence, and toxicity all impact regulation (170). The consequence of a lack of nanomedicine-specific legislation means that such products are regulated like their non-nano counterparts, under existing legislation on medical products and devices (171). While the directives on medical products and medical devices lay down essential requirements for market authorization, these are general and non-specific. As a consequence, the risk assessment, safety, and quality requirements may not be suitable for nanomedicines (172). Concerns about the adequacy of regulatory oversight, in turn, threaten to slow down the development and commercialization of nanomedicine-based products (173), despite the efforts made by the regulatory (FDA and EMA) and consumer agencies to address these matters.

Integration of different academic disciplines with industry and regulatory bodies in a harmonized manner across national and regional boundaries is what is needed most; with all stakeholders working together 
to ensure a well-designed regulatory environment, and to direct future research toward safe, effective nanomedicine products that are translatable and competitive.

In conclusion, it is evident that specific efforts are needed to develop measures for implementing existing regulations that would answer the questions raised by the clinical translation of nanomedicines. These efforts should be scientific, technological, and legislative, and they call for the whole nanomedicine stakeholder community and its professional networks to integrate across Europe first and Internationally. Examples of such efforts include the National Initiatives, the European Technology Platform on Nanomedicine (ETPN), the "Targeted Nano-Pharmaceuticals and Early Diagnostics" cluster, and the European Foundation for Clinical Nanomedicine (CLINAM). Large-scale nanomedicine projects can act as interdisciplinary exchange grounds, connecting academic groups with industrial partners from small enterprises to "big pharma". Knowledge and ideas are shared and expanded among project partners, producing information and technology that can benefit other projects in the field, as well as much larger sections of the stakeholder community. The overall effect is to bring the global health benefits of nanomedicine ever closer. 


\section{References}

1. Morigi V, Tocchio A, Bellavite Pellegrini C, Sakamoto JH, Arnone M, Tasciotti E. Nanotechnology in medicine: from inception to market domination. J Drug Deliv. 2012;2012:389485. PubMed PMID: 22506121. PMCID: 3312282.

2. White Paper to the Horizon 2020 Framework Programme for Research and Innovation Recommendations from the Nanomedicine Community [November 2014]. Available from: http://www.etp-nanomedicine.eu/public/press-documents/publications/etpn-publications/etpn-whitepaper-H2020.

3. Etheridge ML, Campbell SA, Erdman AG, Haynes CL, Wolf SM, McCullough J. The big picture on nanomedicine: the state of investigational and approved nanomedicine products. Nanomedicine. 2013 Jan;9(1):1-14. PubMed PMID: 22684017.

4. Schroeder A, Heller DA, Winslow MM, Dahlman JE, Pratt GW, Langer R, et al. Treating metastatic cancer with nanotechnology. Nat Rev Cancer. 2012 Jan;12(1):39-50. PubMed PMID: 22193407.

5. Ferrari M. Cancer nanotechnology: opportunities and challenges. Nat Rev Cancer. 2005 Mar;5(3):161-71. PubMed PMID: 15738981.

6. Jain KK. Role of nanobiotechnology in the development of personalized medicine. Nanomedicine (Lond). 2009 Apr;4(3):249-52. PubMed PMID: 19331532.

7. Bae KH, Chung HJ, Park TG. Nanomaterials for cancer therapy and imaging. Mol Cells. 2011 Apr;31(4):295-302. PubMed PMID: 21360197.

8. Sakamoto JH, van de Ven AL, Godin B, Blanco E, Serda RE, Grattoni A, et al. Enabling individualized therapy through nanotechnology. Pharmacol Res. 2010 Aug;62(2):57-89. PubMed PMID: 20045055. PMCID: 2886806.

9. Prabhu P, Patravale V. The upcoming field of theranostic nanomedicine: an overview. J Biomed Nanotechnol. 2012 Dec;8(6):859-82. PubMed PMID: 23029995. 
10. Melancon MP, Stafford RJ, Li C. Challenges to effective cancer nanotheranostics. J Control Release. 2012 Dec 10;164(2):177-82. PubMed PMID: 22906841. PMCID: 3504179.

11. Khorasani AA, Weaver JL, Salvador-Morales C. Closing the gap: accelerating the translational process in nanomedicine by proposing standardized characterization techniques. Int J Nanomedicine. 2014;9:5729-51. PubMed PMID: 25525356.

12. Bawa R. FDA and Nanotech: Baby Steps Lead to Regulatory Uncertainty. In: John Wiley \& Sons L, editor. Bio-Nanotechnology: A Revolution in Food, Biomedical and Health Sciences 2013.

13. Eaton MA, Levy L, Fontaine OM. Delivering nanomedicines to patients: A practical guide. Nanomedicine. 2015 May;11(4):983-92. PubMed PMID: 25724929.

14. Villalonga-Barber C, Micha-Screttas M, Steele BR, Georgopoulos A, Demetzos C. Dendrimers as biopharmaceuticals: synthesis and properties. Curr Top Med Chem. 2008;8(14):1294-309. PubMed PMID: 18855710.

15. Clift MJ, Stone V. Quantum dots: an insight and perspective of their biological interaction and how this relates to their relevance for clinical use. Theranostics. 2012;2(7):668-80. PubMed PMID: 22896769. PMCID: 3418927.

16. Chow EK, Zhang XQ, Chen M, Lam R, Robinson E, Huang H, et al. Nanodiamond therapeutic delivery agents mediate enhanced chemoresistant tumor treatment. Sci Transl Med. 2011 Mar 9;3(73):73ra21. PubMed PMID: 21389265.

17. Partha R, Conyers JL. Biomedical applications of functionalized fullerene-based nanomaterials. Int J Nanomedicine. 2009;4:261-75. PubMed PMID: 20011243. PMCID: 2789438.

18. Klumpp C, Kostarelos K, Prato M, Bianco A. Functionalized carbon nanotubes as emerging nanovectors for the delivery of therapeutics. Biochim Biophys Acta. 2006 Mar;1758(3):404-12. PubMed PMID: 16307724. 
19. Cai W, Gao T, Hong H, Sun J. Applications of gold nanoparticles in cancer nanotechnology. Nanotechnol Sci Appl. 2008;1:17-32. PubMed PMID: 24198458. PMCID: 3781768.

20. Lal S, Clare SE, Halas NJ. Nanoshell-enabled photothermal cancer therapy: impending clinical impact. Acc Chem Res. 2008 Dec;41(12):1842-51. PubMed PMID: 19053240.

21. Sawant KK, Dodiya SS. Recent advances and patents on solid lipid nanoparticles. Recent Pat Drug Deliv Formul. 2008;2(2):120-35. PubMed PMID: 19075903.

22. Sapra P, Tyagi P, Allen TM. Ligand-targeted liposomes for cancer treatment. Curr Drug Deliv. 2005 Oct;2(4):369-81. PubMed PMID: 16305440.

23. Allen TM, Cullis PR. Drug delivery systems: entering the mainstream. Science. 2004 Mar 19;303(5665):1818-22. PubMed PMID: 15031496.

24. Kim BY, Rutka JT, Chan WC. Nanomedicine. N Engl J Med. 2010 Dec 16;363(25):2434-43. PubMed PMID: 21158659.

25. Peer D, Karp JM, Hong S, Farokhzad OC, Margalit R, Langer R. Nanocarriers as an emerging platform for cancer therapy. Nat Nanotechnol. 2007 Dec;2(12):751-60. PubMed PMID: 18654426. 26. Bakht MK, Sadeghi M, Pourbaghi-Masouleh M, Tenreiro C. Scope of nanotechnology-based radiation therapy and thermotherapy methods in cancer treatment. Curr Cancer Drug Targets. 2012 Oct;12(8):998-1015. PubMed PMID: 22809233.

27. Jain RK, Stylianopoulos T. Delivering nanomedicine to solid tumors. Nat Rev Clin Oncol. 2010 Nov;7(11):653-64. PubMed PMID: 20838415. PMCID: Pmc3065247. Epub 2010/09/15. eng.

28. Movia D PC, Tran L, Volkov Y, Prina-Mello A. Multilayered nanoparticles for personalized medicine: translation into clinical markets. Handbook of Clinical Nanomedicine: From Bench to Bedside: Pan Stanford Publishing; 2014.

29. Chauhan VP, Jain RK. Strategies for advancing cancer nanomedicine. Nat Mater. 2013 Nov;12(11):958-62. PubMed PMID: 24150413. 
30. Alexis F, Pridgen E, Molnar LK, Farokhzad OC. Factors affecting the clearance and biodistribution of polymeric nanoparticles. Mol Pharm. 2008 Jul-Aug;5(4):505-15. PubMed PMID: 18672949. PMCID: 2663893.

31. Karmali PP, Kotamraju VR, Kastantin M, Black M, Missirlis D, Tirrell M, et al. Targeting of albumin-embedded paclitaxel nanoparticles to tumors. Nanomedicine. 2009 Mar;5(1):73-82. PubMed PMID: 18829396. PMCID: 2824435.

32. Perrault SD, Walkey C, Jennings T, Fischer HC, Chan WC. Mediating tumor targeting efficiency of nanoparticles through design. Nano Lett. 2009 May;9(5):1909-15. PubMed PMID: 19344179. Epub 2009/04/07. eng.

33. Chauhan VP, Martin JD, Liu H, Lacorre DA, Jain SR, Kozin SV, et al. Angiotensin inhibition enhances drug delivery and potentiates chemotherapy by decompressing tumour blood vessels. Nat Commun. 2013;4:2516. PubMed PMID: 24084631. PMCID: 3806395.

34. Cabral H, Matsumoto Y, Mizuno K, Chen Q, Murakami M, Kimura M, et al. Accumulation of sub$100 \mathrm{~nm}$ polymeric micelles in poorly permeable tumours depends on size. Nat Nanotechnol. 2011 Dec;6(12):815-23. PubMed PMID: 22020122. Epub 2011/10/25. eng.

35. Stylianopoulos T, Poh MZ, Insin N, Bawendi MG, Fukumura D, Munn LL, et al. Diffusion of particles in the extracellular matrix: the effect of repulsive electrostatic interactions. Biophys J. 2010 Sep 8;99(5):1342-9. PubMed PMID: 20816045. PMCID: 2931749.

36. Chauhan VP, Stylianopoulos T, Martin JD, Popovic Z, Chen O, Kamoun WS, et al. Normalization of tumour blood vessels improves the delivery of nanomedicines in a size-dependent manner. Nat Nanotechnol. 2012 Jun;7(6):383-8. PubMed PMID: 22484912. PMCID: 3370066.

37. Petros RA, DeSimone JM. Strategies in the design of nanoparticles for therapeutic applications. Nat Rev Drug Discov. 2010 Aug;9(8):615-27. PubMed PMID: 20616808. 
38. Rajendran L, Knolker HJ, Simons K. Subcellular targeting strategies for drug design and delivery. Nat Rev Drug Discov. 2010 Jan;9(1):29-42. PubMed PMID: 20043027.

39. Huang JG, Leshuk T, Gu FX. Emerging nanomaterials for targeting subcellular organelles. Nano Today. 2011;6(5):478-92.

40. Morille M, Passirani C, Vonarbourg A, Clavreul A, Benoit J-P. Progress in developing cationic vectors for non-viral systemic gene therapy against cancer. Biomaterials. 2008;29(24):3477-96.

41. Zuhorn IS, Engberts JB, Hoekstra D. Gene delivery by cationic lipid vectors: overcoming cellular barriers. Eur Biophys J. 2007 Apr;36(4-5):349-62. PubMed PMID: 17019592.

42. Xu Y, Szoka FC. Mechanism of DNA release from cationic liposome/DNA complexes used in cell transfection. Biochemistry. 1996;35(18):5616-23.

43. Howard KA. Delivery of RNA interference therapeutics using polycation-based nanoparticles. Advanced drug delivery reviews. 2009;61(9):710-20.

44. Shmueli RB, Anderson DG, Green JJ. Electrostatic surface modifications to improve gene delivery. Expert opinion on drug delivery. 2010;7(4):535-50.

45. Lungwitz U, Breunig M, Blunk T, Göpferich A. Polyethylenimine-based non-viral gene delivery systems. European Journal of Pharmaceutics and Biopharmaceutics. 2005;60(2):247-66.

46. Walkey $C D$, Chan WC. Understanding and controlling the interaction of nanomaterials with proteins in a physiological environment. Chemical Society Reviews. 2012;41(7):2780-99.

47. Lundqvist M, Stigler J, Elia G, Lynch I, Cedervall T, Dawson KA. Nanoparticle size and surface properties determine the protein corona with possible implications for biological impacts. Proceedings of the National Academy of Sciences. 2008;105(38):14265-70.

48. Dell'Orco D, Lundqvist M, Oslakovic C, Cedervall T, Linse S. Modeling the time evolution of the nanoparticle-protein corona in a body fluid. PLoS One. 2010;5(6):e10949. 
49. Casals E, Pfaller T, Duschl A, Oostingh GJ, Puntes V. Time evolution of the nanoparticle protein corona. ACS nano. 2010;4(7):3623-32.

50. Wolfram J, Yang Y, Shen J, Moten A, Chen C, Shen H, et al. The nano-plasma interface: Implications of the protein corona. Colloids and Surfaces B: Biointerfaces. 2014.

51. Tenzer S, Docter D, Rosfa S, Wlodarski A, Kuharev Jr, Rekik A, et al. Nanoparticle size is a critical physicochemical determinant of the human blood plasma corona: a comprehensive quantitative proteomic analysis. ACS nano. 2011;5(9):7155-67.

52. Hajipour MJ, Laurent S, Aghaie A, Rezaee F, Mahmoudi M. Personalized protein coronas: a "key" factor at the nanobiointerface. Biomaterials Science. 2014.

53. Byrne JD, Betancourt T, Brannon-Peppas L. Active targeting schemes for nanoparticle systems in cancer therapeutics. Advanced drug delivery reviews. 2008;60(15):1615-26.

54. Schäffler M, Sousa F, Wenk A, Sitia L, Hirn S, Schleh C, et al. Blood protein coating of gold nanoparticles as potential tool for organ targeting. Biomaterials. 2014;35(10):3455-66.

55. Chen N, Li Y, Ye Y, Palmisano M, Chopra R, Zhou S. Pharmacokinetics and pharmacodynamics of nab-paclitaxel in patients with solid tumors: Disposition kinetics and pharmacology distinct from solvent-based paclitaxel. The Journal of Clinical Pharmacology. 2014;54(10):1097-107.

56. McKim Jr JM. Building a tiered approach to in vitro predictive toxicity screening: a focus on assays with in vivo relevance. Combinatorial chemistry \& high throughput screening. 2010;13(2):188.

57. Dawidczyk CM, Kim C, Park JH, Russell LM, Lee KH, Pomper MG, et al. State-of-the-art in design rules for drug delivery platforms: lessons learned from FDA-approved nanomedicines. J Control Release. 2014 Aug 10;187:133-44. PubMed PMID: 24874289. PMCID: 4132889.

58. Davis ME, Chen ZG, Shin DM. Nanoparticle therapeutics: an emerging treatment modality for cancer. Nat Rev Drug Discov. 2008 Sep;7(9):771-82. PubMed PMID: 18758474. 
59. Mansour H, Park C, Bawa R. Design and development of approved nanopharmaceutical products. Handbook of Clinical Nanomedicine: From Bench to Bedside R Bawa, G Audette \& I Rubinstein, Eds Singapore: Pan Stanford Publishing Available May. 2014;31:2014.

60. Zamboni WC. Liposomal, nanoparticle, and conjugated formulations of anticancer agents. Clin Cancer Res. 2005 Dec 1;11(23):8230-4. PubMed PMID: 16322279.

61. Gabizon A, Shmeeda H, Barenholz Y. Pharmacokinetics of pegylated liposomal Doxorubicin: review of animal and human studies. Clin Pharmacokinet. 2003;42(5):419-36. PubMed PMID: 12739982. 62. Sanna V, Pala N, Sechi M. Targeted therapy using nanotechnology: focus on cancer. Int J Nanomedicine. 2014;9:467-83. PubMed PMID: 24531078. PMCID: 3896284.

63. Drugs. UFaDA. FDA approved drug products Online Database. Available from: http://www.accessdata.fda.gov/scripts/cder/drugsatfda/index.cfm.

64. Tan S, Li X, Guo Y, Zhang Z. Lipid-enveloped hybrid nanoparticles for drug delivery. Nanoscale. 2013 Feb 7;5(3):860-72. PubMed PMID: 23292080.

65. Waterhouse DN, Tardi PG, Mayer LD, Bally MB. A comparison of liposomal formulations of doxorubicin with drug administered in free form: changing toxicity profiles. Drug Saf. 2001;24(12):90320. PubMed PMID: 11735647.

66. Forssen EA. The design and development of DaunoXome ${ }^{\circledR}$ for solid tumor targeting in vivo. Advanced drug delivery reviews. 1997;24(2):133-50.

67. Allen TM, Martin FJ, editors. Advantages of liposomal delivery systems for anthracyclines. Seminars in oncology; 2004: Elsevier.

68. Fassas A, Anagnostopoulos A. The use of liposomal daunorubicin (DaunoXome) in acute myeloid leukemia. Leukemia \& lymphoma. 2005;46(6):795-802.

69. Wang R, Billone PS, Mullett WM. Nanomedicine in action: an overview of cancer nanomedicine on the market and in clinical trials. Journal of Nanomaterials. 2013;2013:1. 
70. Phuphanich S, Maria B, Braeckman R, Chamberlain M. A pharmacokinetic study of intra-CSF administered encapsulated cytarabine (DepoCyt) for the treatment of neoplastic meningitis in patients with leukemia, lymphoma, or solid tumors as part of a phase III study. J Neurooncol. 2007 Jan;81(2):2018. PubMed PMID: 16941075.

71. Angst MS, Drover DR. Pharmacology of drugs formulated with DepoFoam: a sustained release drug delivery system for parenteral administration using multivesicular liposome technology. Clin Pharmacokinet. 2006;45(12):1153-76. PubMed PMID: 17112293.

72. FDA approves liposomal vincristine (Marqibo) for rare leukemia. Oncology (Williston Park). 2012 Sep;26(9):841. PubMed PMID: 23061340.

73. Silverman JA, Deitcher SR. Marqibo(R) (vincristine sulfate liposome injection) improves the pharmacokinetics and pharmacodynamics of vincristine. Cancer Chemother Pharmacol. 2013 Mar;71(3):555-64. PubMed PMID: 23212117. PMCID: 3579462.

74. Nardin A, Lefebvre ML, Labroquere K, Faure O, Abastado JP. Liposomal muramyl tripeptide phosphatidylethanolamine: Targeting and activating macrophages for adjuvant treatment of osteosarcoma. Curr Cancer Drug Targets. 2006 Mar;6(2):123-33. PubMed PMID: 16529542.

75. Meyers PA. Muramyl tripeptide (mifamurtide) for the treatment of osteosarcoma. Expert Rev Anticancer Ther. 2009 Aug;9(8):1035-49. PubMed PMID: 19671023.

76. Ando K, Mori K, Corradini N, Redini F, Heymann D. Mifamurtide for the treatment of nonmetastatic osteosarcoma. Expert Opin Pharmacother. 2011 Feb;12(2):285-92. PubMed PMID: 21226638. PMCID: 3413631.

77. Anderson P. Liposomal muramyl tripeptide phosphatidyl ethanolamine: ifosfamide-containing chemotherapy in osteosarcoma. Future Oncol. 2006 Jun;2(3):333-43. PubMed PMID: 16787112. 
78. Fogler WE, Fidler IJ. Comparative interaction of free and liposome-encapsulated nor-muramyl dipeptide or muramyl tripeptide phosphatidylethanolamine (3H-labelled) with human blood monocytes. Int J Immunopharmacol. 1987;9(2):141-50. PubMed PMID: 3583507.

79. Gay B, Cardot JM, Schnell C, van Hoogevest P, Gygax D. Comparative pharmacokinetics of free muramyl tripeptide phosphatidyl ethanolamine (MTP-PE) and liposomal MTP-PE. J Pharm Sci. 1993 Oct;82(10):997-1001. PubMed PMID: 8254500.

80. http://clinicaltrials.gov/ct2/home [September 2014].

81. Gradishar WJ, Tjulandin S, Davidson N, Shaw H, Desai N, Bhar P, et al. Phase III trial of nanoparticle albumin-bound paclitaxel compared with polyethylated castor oil-based paclitaxel in women with breast cancer. J Clin Oncol. 2005 Nov 1;23(31):7794-803. PubMed PMID: 16172456.

82. Tallal L, Tan C, Oettgen H, Wollner N, McCarthy M, Helson L, et al. E. coli L-asparaginase in the treatment of leukemia and solid tumors in 131 children. Cancer. 1970 Feb;25(2):306-20. PubMed PMID: 4905155.

83. Jarrar M, Gaynon PS, Periclou AP, Fu C, Harris RE, Stram D, et al. Asparagine depletion after pegylated E. coli asparaginase treatment and induction outcome in children with acute lymphoblastic leukemia in first bone marrow relapse: a Children's Oncology Group study (CCG-1941). Pediatr Blood Cancer. 2006 Aug;47(2):141-6. PubMed PMID: 16425271.

84. Lenz HJ. Management and preparedness for infusion and hypersensitivity reactions. Oncologist. 2007 May;12(5):601-9. PubMed PMID: 17522249.

85. Hoggatt J, Pelus LM. New G-CSF agonists for neutropenia therapy. Expert Opin Investig Drugs. 2014 Jan;23(1):21-35. PubMed PMID: 24073859.

86. Green MD, Koelbl H, Baselga J, Galid A, Guillem V, Gascon P, et al. A randomized double-blind multicenter phase III study of fixed-dose single-administration pegfilgrastim versus daily filgrastim in 
patients receiving myelosuppressive chemotherapy. Ann Oncol. 2003 Jan;14(1):29-35. PubMed PMID: 12488289.

87. Hoggatt J, Tate TA, Pelus LM. Role of lipegfilgrastim in the management of chemotherapyinduced neutropenia. Int J Nanomedicine. 2015;10:2647-52. PubMed PMID: 25878498. PMCID: 4388090.

88. Oerlemans C, Bult W, Bos M, Storm G, Nijsen JF, Hennink WE. Polymeric micelles in anticancer therapy: targeting, imaging and triggered release. Pharm Res. 2010 Dec;27(12):2569-89. PubMed PMID: 20725771. PMCID: 2982955.

89. Hildebrandt B, Wust P, Ahlers O, Dieing A, Sreenivasa G, Kerner T, et al. The cellular and molecular basis of hyperthermia. Crit Rev Oncol Hematol. 2002 Jul;43(1):33-56. PubMed PMID: 12098606.

90. Wust P, Hildebrandt B, Sreenivasa G, Rau B, Gellermann J, Riess H, et al. Hyperthermia in combined treatment of cancer. Lancet Oncol. 2002 Aug;3(8):487-97. PubMed PMID: 12147435.

91. Lammers T, Kiessling F, Hennink WE, Storm G. Drug targeting to tumors: principles, pitfalls and (pre-) clinical progress. J Control Release. 2012 Jul 20;161(2):175-87. PubMed PMID: 21945285.

92. Borsi L, Balza E, Bestagno M, Castellani P, Carnemolla B, Biro A, et al. Selective targeting of tumoral vasculature: comparison of different formats of an antibody (L19) to the ED-B domain of fibronectin. Int J Cancer. 2002 Nov 1;102(1):75-85. PubMed PMID: 12353237.

93. Arap W, Pasqualini R, Ruoslahti E. Cancer treatment by targeted drug delivery to tumor vasculature in a mouse model. Science. 1998 Jan 16;279(5349):377-80. PubMed PMID: 9430587.

94. Ding Y, Li S, Nie G. Nanotechnological strategies for therapeutic targeting of tumor vasculature. Nanomedicine (Lond). 2013 Jul;8(7):1209-22. PubMed PMID: 23837858. 
95. Huang S, Shao K, Liu Y, Kuang Y, Li J, An S, et al. Tumor-targeting and microenvironmentresponsive smart nanoparticles for combination therapy of antiangiogenesis and apoptosis. ACS Nano. 2013 Mar 26;7(3):2860-71. PubMed PMID: 23451830.

96. Xie J, Shen Z, Li KC, Danthi N. Tumor angiogenic endothelial cell targeting by a novel integrintargeted nanoparticle. Int J Nanomedicine. 2007;2(3):479-85. PubMed PMID: 18019845. PMCID: 2676654.

97. Danhier F, Vroman B, Lecouturier N, Crokart N, Pourcelle V, Freichels H, et al. Targeting of tumor endothelium by RGD-grafted PLGA-nanoparticles loaded with paclitaxel. J Control Release. 2009 Dec 3;140(2):166-73. PubMed PMID: 19699245.

98. Dubey PK, Singodia D, Verma RK, Vyas SP. RGD modified albumin nanospheres for tumour vasculature targeting. J Pharm Pharmacol. 2011 Jan;63(1):33-40. PubMed PMID: 21155813.

99. Tinkle S, McNeil SE, Muhlebach S, Bawa R, Borchard G, Barenholz YC, et al. Nanomedicines: addressing the scientific and regulatory gap. Ann N Y Acad Sci. 2014 Apr;1313:35-56. PubMed PMID: 24673240.

100. Libutti SK, Paciotti GF, Byrnes AA, Alexander HR, Jr., Gannon WE, Walker M, et al. Phase I and pharmacokinetic studies of CYT-6091, a novel PEGylated colloidal gold-rhTNF nanomedicine. Clin Cancer Res. 2010 Dec 15;16(24):6139-49. PubMed PMID: 20876255. PMCID: 3004980.

101. http://www.cytimmune.com [November 2014].

102. Schmitt-Sody M, Strieth S, Krasnici S, Sauer B, Schulze B, Teifel M, et al. Neovascular targeting therapy: paclitaxel encapsulated in cationic liposomes improves antitumoral efficacy. Clin Cancer Res. 2003 Jun;9(6):2335-41. PubMed PMID: 12796403.

103. Thurston G, McLean JW, Rizen M, Baluk P, Haskell A, Murphy TJ, et al. Cationic liposomes target angiogenic endothelial cells in tumors and chronic inflammation in mice. Journal of clinical investigation. 1998;101(7):1401. 
104. Löhr M, Bodoky G, Fölsch U, Märten A, Karrasch M, Lilla C, et al. Cationic liposomal paclitaxel in combination with gemcitabine in patients with advanced pancreatic cancer: a phase II trial. J Clin Oncol. 2009;27(15s):208s.

105. Awada A, Bondarenko I, Tarasova O, Bonneterre J, Nowara E, Ferrero J, et al. Results of the first randomized phase II study of Cationic Liposomal Paclitaxel (EndoTAGTM-1) targeting tumor endothelial cells in advanced triple-negative breast cancer (TNBC). Ann Oncol. 2010;21(8s):viii5.

106. Ribeiro JT, Macedo LT, Curigliano G, Fumagalli L, Locatelli M, Dalton M, et al. Cytotoxic drugs for patients with breast cancer in the era of targeted treatment: back to the future? Ann Oncol. 2012 Mar;23(3):547-55. PubMed PMID: 21896541.

107. http://www.medigene.com [November 2014].

108. O’Brien ME, Socinski MA, Popovich AY, Bondarenko IN, Tomova A, Bilynsky BT, et al.

Randomized phase III trial comparing single-agent paclitaxel Poliglumex (CT-2103, PPX) with single-agent gemcitabine or vinorelbine for the treatment of PS 2 patients with chemotherapy-naive advanced nonsmall cell lung cancer. Journal of Thoracic Oncology. 2008;3(7):728-34.

109. Sabbatini P, Sill MW, O'Malley D, Adler L, Secord AA. A phase II trial of paclitaxel poliglumex in recurrent or persistent ovarian or primary peritoneal cancer (EOC): a Gynecologic Oncology Group Study. Gynecologic oncology. 2008;111(3):455-60.

110. Lin NU, Parker LM, Come SE, Burstein HJ, Haldoupis M, Ryabin N, et al. Phase II study of CT-2103 as first-or second-line chemotherapy in patients with metastatic breast cancer: unexpected incidence of hypersensitivity reactions. Investigational new drugs. 2007;25(4):369-75.

111. Treat J, Damjanov N, Huang C, Zrada S, Rahman A. Liposomal-encapsulated chemotherapy: preliminary results of a phase I study of a novel liposomal paclitaxel. Oncology (Williston Park, NY). 2001;15(5 Suppl 7):44-8. 
112. Newman MS, Colbern GT, Working PK, Engbers C, Amantea MA. Comparative pharmacokinetics, tissue distribution, and therapeutic effectiveness of cisplatin encapsulated in long-circulating, pegylated liposomes (SPI-077) in tumor-bearing mice. Cancer Chemother Pharmacol. 1999 1999/01/01;43(1):1-7. English.

113. Harrington KJ, Lewanski CR, Northcote AD, Whittaker J, Wellbank H, Vile RG, et al. Phase I-II study of pegylated liposomal cisplatin (SPI-077 TM) in patients with inoperable head and neck cancer. Annals of Oncology. 2001 April 1, 2001;12(4):493-6.

114. SEETHARAMU N, KIM E, HOCHSTER H, MARTIN F, MUGGIA F. Phase II Study of Liposomal Cisplatin (SPI-77) in Platinum-sensitive Recurrences of Ovarian Cancer. Anticancer Research. 2010 February 1, 2010;30(2):541-5.

115. Seetharamu N, Kim E, Hochster H, Martin F, Muggia F. Phase Il study of liposomal cisplatin (SPI77) in platinum-sensitive recurrences of ovarian cancer. Anticancer Res. 2010 Feb;30(2):541-5. PubMed PMID: 20332467.

116. White SC, Lorigan P, Margison GP, Margison JM, Martin F, Thatcher N, et al. Phase II study of SPI-77 (sterically stabilised liposomal cisplatin) in advanced non-small-cell lung cancer. Br J Cancer. 2006 Oct 9;95(7):822-8. PubMed PMID: 16969346. PMCID: 2360546.

117. Veal GJ, Griffin MJ, Price E, Parry A, Dick GS, Little MA, et al. A phase I study in paediatric patients to evaluate the safety and pharmacokinetics of SPI-77, a liposome encapsulated formulation of cisplatin. Br J Cancer. 2001 Apr 20;84(8):1029-35. PubMed PMID: 11308249. PMCID: 2363870.

118. Harrington KJ, Lewanski CR, Northcote AD, Whittaker J, Wellbank H, Vile RG, et al. Phase I-II study of pegylated liposomal cisplatin (SPI-077) in patients with inoperable head and neck cancer. Ann Oncol. 2001 Apr;12(4):493-6. PubMed PMID: 11398881. 
119. Kim ES, Lu C, Khuri FR, Tonda M, Glisson BS, Liu D, et al. A phase Il study of STEALTH cisplatin (SPI-77) in patients with advanced non-small cell lung cancer. Lung Cancer. 2001 Dec;34(3):427-32. PubMed PMID: 11714540.

120. Rosenthal DI, Yom SS, Liu L, Machtay M, Algazy K, Weber RS, et al. A phase I study of SPI-077 (Stealth liposomal cisplatin) concurrent with radiation therapy for locally advanced head and neck cancer. Invest New Drugs. 2002 Aug;20(3):343-9. PubMed PMID: 12201498.

121. Vokes EE, Gordon GS, Mauer AM, Rudin CM, Krauss SA, Szeto L, et al. A phase I study of STEALTH cisplatin (SPI-77) and vinorelbine in patients with advanced non small-cell lung cancer. Clin Lung Cancer. 2000 Nov;2(2):128-32. PubMed PMID: 14731323.

122. Oberoi HS, Nukolova NV, Kabanov AV, Bronich TK. Nanocarriers for delivery of platinum anticancer drugs(). Advanced drug delivery reviews. 2013 10/08;65(0):1667-85. PubMed PMID: PMC4197009.

123. Valle JW, Armstrong A, Newman C, Alakhov V, Pietrzynski G, Brewer J, et al. A phase 2 study of SP1049C, doxorubicin in P-glycoprotein-targeting pluronics, in patients with advanced adenocarcinoma of the esophagus and gastroesophageal junction. Invest New Drugs. 2011 Oct;29(5):1029-37. PubMed PMID: 20179989.

124. Matsumura Y, Hamaguchi T, Ura T, Muro K, Yamada Y, Shimada Y, et al. Phase I clinical trial and pharmacokinetic evaluation of NK911, a micelle-encapsulated doxorubicin. Br J Cancer. 2004 Nov 15;91(10):1775-81. PubMed PMID: 15477860. PMCID: 2410050.

125. Endo K, Ueno T, Kondo S, Wakisaka N, Murono S, Ito M, et al. Tumor-targeted chemotherapy with the nanopolymer-based drug NC-6004 for oral squamous cell carcinoma. Cancer Sci. 2013 Mar;104(3):369-74. PubMed PMID: 23216802.

126. Weiss GJ, Chao J, Neidhart JD, Ramanathan RK, Bassett D, Neidhart JA, et al. First-in-human phase $1 / 2$ a trial of CRLX101, a cyclodextrin-containing polymer-camptothecin nanopharmaceutical in 
patients with advanced solid tumor malignancies. Invest New Drugs. 2013 Aug;31(4):986-1000. PubMed PMID: 23397498. PMCID: 3774600.

127. Kono K, Nakashima S, Kokuryo D, Aoki I, Shimomoto H, Aoshima S, et al. Multi-functional liposomes having temperature-triggered release and magnetic resonance imaging for tumor-specific chemotherapy. Biomaterials. 2011 Feb;32(5):1387-95. PubMed PMID: 21093041.

128. Poon RT, Borys N. Lyso-thermosensitive liposomal doxorubicin: a novel approach to enhance efficacy of thermal ablation of liver cancer. Expert Opin Pharmacother. 2009 Feb;10(2):333-43. PubMed PMID: 19236203.

129. Dromi S, Frenkel V, Luk A, Traughber B, Angstadt M, Bur M, et al. Pulsed-high intensity focused ultrasound and low temperature-sensitive liposomes for enhanced targeted drug delivery and antitumor effect. Clin Cancer Res. 2007 May 1;13(9):2722-7. PubMed PMID: 17473205. PMCID: 2555974.

130. Johannsen $M$, Thiesen $B$, Wust $P$, Jordan A. Magnetic nanoparticle hyperthermia for prostate cancer. Int J Hyperthermia. 2010;26(8):790-5. PubMed PMID: 20653418.

131. Dennis CL, Jackson AJ, Borchers JA, Hoopes PJ, Strawbridge R, Foreman AR, et al. Nearly complete regression of tumors via collective behavior of magnetic nanoparticles in hyperthermia. Nanotechnology. 2009 Sep 30;20(39):395103. PubMed PMID: 19726837.

132. Gad SC, Sharp KL, Montgomery C, Payne JD, Goodrich GP. Evaluation of the toxicity of intravenous delivery of auroshell particles (gold-silica nanoshells). Int J Toxicol. 2012 Nov-Dec;31(6):58494. PubMed PMID: 23212452.

133. Schwartz JA, Shetty AM, Price RE, Stafford RJ, Wang JC, Uthamanthil RK, et al. Feasibility study of particle-assisted laser ablation of brain tumors in orthotopic canine model. Cancer Res. 2009 Feb 15;69(4):1659-67. PubMed PMID: 19208847. 
134. Rivera Gil P, Huhn D, del Mercato LL, Sasse D, Parak WJ. Nanopharmacy: Inorganic nanoscale devices as vectors and active compounds. Pharmacol Res. 2010 Aug;62(2):115-25. PubMed PMID: 20097288.

135. Shi J, Xiao Z, Kamaly N, Farokhzad OC. Self-assembled targeted nanoparticles: evolution of technologies and bench to bedside translation. Acc Chem Res. 2011 Oct 18;44(10):1123-34. PubMed PMID: 21692448.

136. Qian ZM, Li H, Sun H, Ho K. Targeted drug delivery via the transferrin receptor-mediated endocytosis pathway. Pharmacol Rev. 2002 Dec;54(4):561-87. PubMed PMID: 12429868.

137. Wang S, Low PS. Folate-mediated targeting of antineoplastic drugs, imaging agents, and nucleic acids to cancer cells. J Control Release. 1998 Apr 30;53(1-3):39-48. PubMed PMID: 9741912.

138. Davis ME, Zuckerman JE, Choi CH, Seligson D, Tolcher A, Alabi CA, et al. Evidence of RNAi in humans from systemically administered siRNA via targeted nanoparticles. Nature. $2010 \mathrm{Apr}$ 15;464(7291):1067-70. PubMed PMID: 20305636. PMCID: 2855406.

139. Zuckerman JE, Gritli I, Tolcher A, Heidel JD, Lim D, Morgan R, et al. Correlating animal and human phase la/Ib clinical data with CALAA-01, a targeted, polymer-based nanoparticle containing siRNA. Proceedings of the National Academy of Sciences. 2014 August 5, 2014;111(31):11449-54. 140. Gu F, Zhang L, Teply BA, Mann N, Wang A, Radovic-Moreno AF, et al. Precise engineering of targeted nanoparticles by using self-assembled biointegrated block copolymers. Proc Natl Acad Sci U S A. 2008 Feb 19;105(7):2586-91. PubMed PMID: 18272481. PMCID: 2268180.

141. Venditto VJ, Szoka Jr FC. Cancer nanomedicines: so many papers and so few drugs! Advanced drug delivery reviews. 2013;65(1):80-8.

142. Valencia PM, Farokhzad OC, Karnik R, Langer R. Microfluidic technologies for accelerating the clinical translation of nanoparticles. Nat Nanotechnol. 2012 Oct;7(10):623-9. PubMed PMID: 23042546. PMCID: 3654404. 
143. Ehmann F, Sakai-Kato K, Duncan R, Hernan Perez de la Ossa D, Pita R, Vidal JM, et al. Nextgeneration nanomedicines and nanosimilars: EU regulators' initiatives relating to the development and evaluation of nanomedicines. Nanomedicine (Lond). 2013 May;8(5):849-56. PubMed PMID: 23656268. 144. Nystrom AM, Fadeel B. Safety assessment of nanomaterials: implications for nanomedicine. J Control Release. 2012 Jul 20;161(2):403-8. PubMed PMID: 22306428.

145. Fadeel B, Garcia-Bennett AE. Better safe than sorry: Understanding the toxicological properties of inorganic nanoparticles manufactured for biomedical applications. Adv Drug Deliv Rev. 2010 Mar 8;62(3):362-74. PubMed PMID: 19900497.

146. Murday JS, Siegel RW, Stein J, Wright JF. Translational nanomedicine: status assessment and opportunities. Nanomedicine. 2009 Sep;5(3):251-73. PubMed PMID: 19540359.

147. Stone V, Johnston H, Schins RP. Development of in vitro systems for nanotoxicology: methodological considerations. Crit Rev Toxicol. 2009;39(7):613-26. PubMed PMID: 19650720. 148. Cho EC, Zhang Q, Xia Y. The effect of sedimentation and diffusion on cellular uptake of gold nanoparticles. Nature nanotechnology. 2011 Jun;6(6):385-91. PubMed PMID: 21516092. PMCID: 3227810. Epub 2011/04/26. eng.

149. Napierska D, Thomassen LC, Lison D, Martens JA, Hoet PH. The nanosilica hazard: another variable entity. Part Fibre Toxicol. 2010;7(1):39. PubMed PMID: 21126379. PMCID: 3014868.

150. Vinci M, Gowan S, Boxall F, Patterson L, Zimmermann M, Court W, et al. Advances in establishment and analysis of three-dimensional tumor spheroid-based functional assays for target validation and drug evaluation. BMC biology. 2012;10:29. PubMed PMID: 22439642. PMCID: 3349530. Epub 2012/03/24. eng.

151. Nyga A, Cheema U, Loizidou M. 3D tumour models: novel in vitro approaches to cancer studies. Journal of cell communication and signaling. 2011 Aug;5(3):239-48. PubMed PMID: 21499821. PMCID: 3145874. Epub 2011/04/19. eng. 
152. Cho EJ, Holback H, Liu KC, Abouelmagd SA, Park J, Yeo Y. Nanoparticle characterization: state of the art, challenges, and emerging technologies. Mol Pharm. 2013 Jun 3;10(6):2093-110. PubMed PMID: 23461379. PMCID: 3672343.

153. Nel AE, Madler L, Velegol D, Xia T, Hoek EM, Somasundaran P, et al. Understanding biophysicochemical interactions at the nano-bio interface. Nat Mater. 2009 Jul;8(7):543-57. PubMed PMID: 19525947.

154. Desai N. Challenges in Development of Nanoparticle-Based Therapeutics. AAPS J. 2012 2012/06/01;14(2):282-95. English.

155. Stern ST, Hall JB, Yu LL, Wood LJ, Paciotti GF, Tamarkin L, et al. Translational considerations for cancer nanomedicine. Journal of Controlled Release. 2010 9/1/;146(2):164-74.

156. Hoet $P$, Legiest B, Geys J, Nemery B. Do nanomedicines require novel safety assessments to ensure their safety for long-term human use? Drug Saf. 2009;32(8):625-36. PubMed PMID: 19591528. 157. Stern ST, Hall JB, Yu LL, Wood LJ, Paciotti GF, Tamarkin L, et al. Translational considerations for cancer nanomedicine. J Control Release. 2010 Sep 1;146(2):164-74. PubMed PMID: 20385183. PMCID: 2921639.

158. Crist RM, Grossman JH, Patri AK, Stern ST, Dobrovolskaia MA, Adiseshaiah PP, et al. Common pitfalls in nanotechnology: lessons learned from NCl's Nanotechnology Characterization Laboratory. Integrative Biology. 2013;5(1):66-73.

159. Muthu MS, Wilson B. Challenges posed by the scale-up of nanomedicines. Nanomedicine. 2012 2012/03/01;7(3):307-9.

160. Draft Guidance for Industry: Considering Whether an FDA Regulated Product Involves the Application of Nanotechnology 2011. Available from: http://www.fda.gov/Regulatorylnformation/Guidances/ucm257698.htm. 
161. Commission Recommendation of 18 October 2011 on the definition of nanomaterial [November 2014]. Available from:

http://eurlex.europa.eu/LexUriServ/LexUriServ.do?uri=OJ:L:2011:275:0038:0040:EN:PDF.

162. Scientific Committee on Emerging and Newly Identified Health Risks. Scientific Basis for the Definition of the Term "nanomaterial". 2010. Available from:

http://ec.europa.eu/health/scientific committees/emerging/docs/scenihr o 030.pdf.

163. Sparreboom A, Scripture CD, Trieu V, Williams PJ, De T, Yang A, et al. Comparative preclinical and clinical pharmacokinetics of a cremophor-free, nanoparticle albumin-bound paclitaxel (ABI-007) and paclitaxel formulated in Cremophor (Taxol). Clin Cancer Res. 2005 Jun 1;11(11):4136-43. PubMed PMID: 15930349.

164. Commission E. Proposal for a regulation of the European Parliament and of the Council on medical devices, and amending Directive 2001/83/EC, Regulation (EC) No 178/2002 and Regulation (EC) No 1223/2009 2012. Available from: http://ec.europa.eu/health/medicaldevices/files/revision docs/proposal 2012542 en.pdf.

165. Hafner A, Lovrić J, Lakoš GP, Pepić I. Nanotherapeutics in the EU: an overview on current state and future directions. International journal of nanomedicine. 2014;9:1005.

166. Administration UFaD. Nanotechnology: a report of the US Food and Drug Administration, Nanotechnology Task Force. Rockville, MD: US Food and Drug Administration. 2007.

167. Food U, Administration D. Draft Guidance for Industry: Considering Whether an FDA-Regulated Product Involves the Application of Nanotechnology. US FDA. 2011.

168. Hamburg MA. FDA's Approach to Regulation of Products of Nanotechnology. Science. 2012;336(6079):299-300.

169. Milmo S. Seeking Harmonization in Nanomedicines Regulatory Framework BioPharm International 2013;26(8):12-3. 
170. Dorbeck-Jung BR CN. Is the European Medical Products Authorisation Regulation Equipped to Cope with the Challenges of Nanomedicines? Law \& Policy. 2011;3(2):276-303.

171. Geert van Calster. Regulating nanotechnology in the European Union Nanotechnology, Law and Business. 2006;3(3):359-72.

172. Toma-Bianov A. Europena Union regulations on Nanomedicine. Bulletin of the Transilvania University of Braşov Series VII: Social Sciences. 2012;5 (54)(2).

173. D'Silva J, Calster Gv. Taking Temperature - A Review of European Union Regulation in Nanomedicine. European Journal of Health Law. 2009;16:249-69. 


\section{Table legends:}

Table 1. Clinically approved nanomedicines for cancer treatment. Sources: www.accessdata.fda.org; http://www.ema.europa.eu/ema/, accessed on June $26^{\text {th }} 2015$.

Table 2. Candidate oncological nanomedicines currently undergoing clinical trials. Nanomaterial groupings were chosen for consistency with Table 1. Unless specified, the information source is the US National Institutes of Health clinical trials website (http://clinicaltrials.gov/ct2/home), accessed on June $26^{\text {th }}$ 2015. Recruiting, active, or completed trials are listed; terminated or not yet recruiting trials are not included.

Table 3. EMA reflection papers on nanomedicine. Source: http://www.ema.europa.eu/ema .

\section{Figure legends}

Figure 1. Multiple disciplines converge in cancer nanomedicine. As shown, the field requires integration of knowledge from many scientific disciplines.

Figure 2. Stakeholders and Challenges. The main nanomedicine stakeholders (i.e., clinicians, patients, industry, regulatory bodies, and academic researchers) and the main challenges faced in the translation of nanomedicines.

Figure 3. Schematic representation of a typical cancer nanomedicine's journey through the body, highlighting the importance of its targeted physico-chemical properties. These properties strongly 
influence the transport, distribution, efficacy, toxicity, and persistence of the nanomedicine in the patient. Nanomedicine design requires understanding of the interactions between the nanomaterial and all the different physiological compartments it will encounter during and after administration.

Figure 4. Delivery strategies, physiology-based design strategies and next-generation challenges for different cancer nanomedicine platforms. Liposomes are being developed for all delivery strategies (passive and active targeting of tumor or endothelial cells, and triggered drug delivery). Micelles are mainly being developed for passive delivery of drugs, while polymeric nanoparticles are being used for passive and active delivery. Metal based nanoparticles are mainly being developed for passive delivery and triggered drug delivery (thermaltherapy). All platforms enhance blood stability and longer elimination time compared to coventional drugs, whereas intratumor accumulation and drug/carrier ratio are superior for liposomes and polymeric nanoparticles. Orange boxes highlight the design challenges facing designers of the next-generation of cancer nanomedicines.

Fig 5: Publication trend on the topic of "nanotechnology for treatment of cancer". Two public databases were searched for "nanotechnology AND cancer AND treatment", sorted by year of publication from 2004 to 2013. A: Pubmed medical sciences database (http://www.ncbi.nlm.nih.gov/pubmed) displayed a total of about 2,500 papers, with about 800 of those being reviews (orange bars) and the remaining majority being original-research papers (blue bars). B: Google scholar (scholar.google.com) searches many scientific databases and provides a more extensive list of publications. With the exclusion of patents and citations, it displayed a total of almost 65,000 publications within the same period. 